\title{
STOCHASTIC OPTIMAL CONTROL OF A EVOLUTIONARY $p$-LAPLACE EQUATION WITH MULTIPLICATIVE LÉVY NOISE.
}

\author{
ANANTA K. MAJEE
}

\begin{abstract}
In this article, we are interested in an initial value optimal control problem for a evolutionary $p$-Laplace equation driven by multiplicative Lévy noise. We first present wellposedness of a weak solution by using an implicit time discretization of the problem, along with the Jakubowski version of the Skorokhod theorem for a non-metric space. We then formulate associated control problem, and establish existence of an optimal solution by using variational method and exploiting the convexity property of the cost functional.
\end{abstract}

\section{INTRODUCTION}

The last couple of decades have witnessed remarkable advances on the larger area of stochastic partial differential equations that are driven by Lévy noise. An worthy reference on this subject is [25]. In this article, we are interested in the specific problem of evolution equation with Lévy noise, and aim to prove existence of a weak optimal solution of an initial value control evolutionary $p$-Laplace equation driven by Lévy noise. A formal description of our problem as follows. Let $\left(\Omega, \mathcal{F}, \mathbb{P},\left\{\mathcal{F}_{t}\right\}_{t \geq 0}\right)$ be a filtered probability space satisfying the usual hypotheses i.e. $\left\{\mathcal{F}_{t}\right\}_{t \geq 0}$ is a right-continuous filtration such that $\mathcal{F}_{0}$ contains all the $\mathbb{P}$-null subsets of $(\Omega, \mathcal{F})$. In addition, let $N(\mathrm{~d} z, \mathrm{~d} t)$ be a time homogeneous Poisson random measure 1 on $\mathbb{R}$ with intensity measure $m(\mathrm{~d} z)$ with respect to the same stochastic basis. We are interested in the initial value control evolution equation of the type

$$
\begin{aligned}
\mathrm{d} u-\operatorname{div}_{x}\left(|\nabla u|^{p-2} \nabla u+\vec{f}(u)\right) \mathrm{d} t & =\int_{|z|>0} \eta(u ; z) \tilde{N}(d z, \mathrm{~d} t) \quad \text { in } \Omega \times D_{T}, \\
u & =0 \text { on } \Omega \times \partial D_{T}, \\
u(0, \cdot) & =u_{0}(\cdot)+U(\cdot) \quad \text { in } \Omega \times D,
\end{aligned}
$$

where $p>2, D_{T}=(0, T) \times D$ with $T>0$ fixed, $D \subset \mathbb{R}^{d}$ is a bounded domain with Lipschitz boundary $\partial D, \partial D_{T}=(0, T) \times \partial D, u$ is the unknown random scalar valued function, $\vec{f}: \mathbb{R} \rightarrow \mathbb{R}^{d}$ is a given flux function, and $\widetilde{N}(\mathrm{~d} z, \mathrm{~d} t)=N(\mathrm{~d} z, \mathrm{~d} t)-m(\mathrm{~d} z) \mathrm{d} t$, the compensated time homogeneous Poisson random measure. Furthermore, $(u, z) \mapsto \eta(u ; z)$ is a real valued function defined on the domain $\mathbb{R} \times \mathbb{R}$. The stochastic integral in the right hand side of (1.1) is defined in the Lévy-Itô sense.

We point out that adding a Brownian component to the Lévy noise term on the right hand side of (1.1) would make it more general, and the results of this paper are still valid under appropriate conditions.

The equation (1.1) could be viewed as a stochastic perturbation of a evolution $p$-Laplacian equation with nonlinear sources. Equations of this type arise in the field of mechanics, physics and biology [8, 31]. In the case $\eta=0$, the equation (1.1) becomes a deterministic evolution

2000 Mathematics Subject Classification. 45K05, 46S50, 49L20, 49L25, 91A23, 93E20.

Key words and phrases. Evolutionary $p$-Laplace equation, Stochastic PDEs, Weak solution, Skorokhod theorem .

${ }^{1}$ For the definition of a time homogeneous Poisson random measure, we refer to see [4, pp. 631]. 
$p$-Laplacian equation with nonlinear sources, and there is a plethora literature (see [20, 32] and references therein) for its wellposedness.

Due to more technical novelties, the study of wellposedness result in case of nonlinear $p$ evolutionary equation with nonlinear stochastic forcing is more subtle. The presence of nonlinearity in the drift and diffusion terms in equation prevents us to define a semi-group solution. Moreover, because of nonlinear perturbation $\operatorname{div}_{x} f(u)$ of $p$-Laplace operator $(p>2)$, one can not use the results of monotone or locally monotone SPDEs, see e.g., [15, 23. In a recent article [28, the authors have considered (1.1) with cylindrical Wiener process $W=\left\{W_{t}: t \in[0, T]\right\}$ in $L^{2}(D)$, and proved wellposedness of strong solution. In [28], existence of a martingale solution is shown by constructing an approximate solution (via implicit time discretization) and deriving its a-priori estimates which are used to apply Jakubowski-Skorokhod theorem in a non-metric space. Then, using an argument of path-wise uniqueness and Gyöngy-Krylov characterization [12] of convergence in probability, the authors established wellposedness of strong solution.

In this paper, our goal is to find a weak admissible solution $\pi^{*}:=\left(\Omega^{*}, \mathcal{F}^{*}, \mathbb{P}^{*},\left\{\mathcal{F}_{t}^{*}\right\}, N^{*}, u^{*}, U^{*}\right)$ which minimizes

$$
\begin{array}{r}
\mathcal{J}(\pi)=\mathbb{E}\left[\int_{0}^{T}\left\|u(t)-u_{\operatorname{tar}}(t)\right\|_{L^{2}(D)}^{2} \mathrm{~d} t+\|U\|_{W^{1, p}}^{p}+\Psi(u(T))\right] \\
\text { with } \pi=\left(\Omega, \mathcal{F}, \mathbb{P},\left\{\mathcal{F}_{t}\right\}, N, u, U\right) \text { subject to (1.1) },
\end{array}
$$

for a given deterministic target profile $u_{\mathrm{tar}}$, and terminal payoff $\Psi$. The existing literature (see e.g. [21]) on stochastic optimal control with SPDEs mainly considers those which has a mild solution, which is not available for problem (1.1). For this reason, we use variational method to construct a minimizer $\pi^{*}$ of (1.2), see also [7, 9]. Being motivated from [7, 9, 28, our aim is twofold:

i) Firstly, we prove existence of a weak solution of the problem (1.1). We construct an approximate solutions $\tilde{u}_{\Delta t}:=\left\{\tilde{u}_{\Delta t}(t) ; t \in[0, T]\right\}$ (cf. (3.5)) via implicit time discretization, and derive its a-priori bounds which is used to show the tightness of the laws of sequence $\left(\tilde{u}_{\Delta t}\right)$, denoted by $\mathcal{L}\left(\tilde{u}_{\Delta t}\right)$, in some appropriate space via Aldous condition (see Definition [3.1). We then use the Jakubowski version of the Skorokhod theorem in a non-metric space to show existence of a weak solution of (1.1). We use smooth approximation of absolute value function and then apply Itô-Lévy formula, and pass to the limit as approximation parameter goes to zero to show the path-wise uniqueness of weak solutions.

ii) Secondly, we construct a minimizer $\pi^{*}$ of the control problem (1.2) by considering a minimizing weak admissible solutions $\pi_{n}=\left(\Omega_{n}, \mathcal{F}_{n}, \mathbb{P}_{n},\left\{\mathcal{F}_{t}^{n}\right\}, N_{n}, u_{n}, U_{n}\right)$ along with Skorokhod's theorem and exploiting the convexity property of the cost functional $\mathcal{J}$ with respect to the control variable.

The remaining of the paper is organized as follows. We state the assumptions, detail the technical framework and state the main results in Section 2. In Section 3, we construct an approximate solutions, derive its a-priori estimates, show the tightness of the laws of the approximate solutions in some space, and then apply the Jakubowski version of the Skorokhod theorem to have a existence of a weak solution of the problem. Moreover, path-wise uniqueness of weak solutions is shown in Subsection 3.5.1. The final section is devoted to establish existence of an optimal solution of the initial value control problem (1.2).

\section{TeChNicAl FRAmeWork AND STATEMENT OF the MAIN RESUlts}

Throughout this paper, we use the letter $C$ to denote various generic constants. In the sequel, we denote by $\langle\cdot, \cdot\rangle$, the pairing between $W_{0}^{1, p}(D)$ and $W^{-1, p^{\prime}}(D)$ where $p^{\prime}$ denotes the convex conjugate of $p$. 
In the theory of stochastic evolution equations, two types of solution concept are considered namely strong solution and weak solution. A strong solution is typically an analytically weak solution (in space) on a given stochastic basis. In general, for a nonlinear non-Lipschitz drift operator, one may not able to prove existence of a strong solution, and therefore needs to consider concept of weak solution.

Definition 2.1. (Weak solution) A weak solution of (1.1) is a 7-tuple $\bar{\pi}=\left(\bar{\Omega}, \overline{\mathcal{F}}, \overline{\mathbb{P}},\left\{\overline{\mathcal{F}}_{t}\right\}, \bar{N}, \bar{u}, \bar{U}\right)$ such that

i) $(\bar{\Omega}, \overline{\mathcal{F}}, \overline{\mathbb{P}})$ is a complete probability space endowed with the filtration $\left\{\overline{\mathcal{F}}_{t}\right\}$ satisfying the usual hypotheses.

ii) $\bar{N}$ is a time-homogeneous Poisson random measure on $\mathbb{R}$ with intensity measure $m(d z)$ with respect to the filtration $\left\{\overline{\mathcal{F}}_{t}\right\}$.

iii) $\bar{U}$ is measurable with $\overline{\mathbb{P}}$-a.s. $\omega \in \bar{\Omega}, \bar{U}(\omega, \cdot) \in W^{1, p}(D)$.

iv) $\bar{u}: \bar{\Omega} \times[0, T] \rightarrow L^{2}(D)$ is an $W_{0}^{1, p}(D)$-valued $\left\{\overline{\mathcal{F}}_{t}\right\}$-predictable stochastic process such that $\overline{\mathbb{P}}$-a.s.,

a) $\bar{u} \in L^{p}\left(0, T ; W_{0}^{1, p}(D)\right) \cap L^{\infty}\left(0, T ; L^{2}(D)\right)$ and $\bar{u}(0, \cdot)=u_{0}+\bar{U}$ in $L^{2}(D)$.

b) for all $t \in[0, T]$, there holds

$$
\bar{u}(t)=u_{0}+\bar{U}+\int_{0}^{t} \operatorname{div}_{x}\left(|\nabla \bar{u}|^{p-2} \nabla \bar{u}+\vec{f}(\bar{u})\right) \mathrm{d} s+\int_{0}^{t} \int_{|z|>0} \eta(\bar{u} ; z) \widetilde{\bar{N}}(\mathrm{~d} z, \mathrm{~d} s) \quad \text { in } \quad L^{2}(D) .
$$

We show the wellposedness of weak solution of (1.1), in the sense of Definition 2.1, under the following assumptions:

A.1 $u_{0} \in L^{2}(D)$.

A.2 $\vec{f}: \mathbb{R} \mapsto \mathbb{R}^{d}$ is $C^{2}$ and Lipschitz continuous with $\vec{f}(0)=0$.

A.3 $\eta(0 ; z)=0$ for all $z \in \mathbb{R}$. Moreover, there exists positive constant $0<\lambda^{*}<1$ such that 2 for all $u, v \in \mathbb{R}$ and $z \in \mathbb{R}$

$$
|\eta(u ; z)-\eta(v ; z)| \leq \lambda^{*}|u-v|(1 \wedge|z|) .
$$

A.4 The Lévy measure $m(\mathrm{~d} z)$ is a Radon measure on $\mathbb{R} \backslash\{0\}$ with a possible singularity at $z=0$, which satisfies

$$
c_{\eta}:=\int_{|z|>0}\left(1 \wedge|z|^{2}\right) m(\mathrm{~d} z)<+\infty .
$$

Theorem 2.1. Let the assumptions A.1 A.4 be true. Let $\left(\Omega, \mathcal{F}, \mathbb{P},\left\{\mathcal{F}_{t}\right\}\right)$ be a given filtered probability space satisfying the usual hypotheses and $N$ be a time-homogeneous Poisson random measure on $\mathbb{R}$ with intensity measure $m(\mathrm{~d} z)$ defined on $\left(\Omega, \mathcal{F}, \mathbb{P},\left\{\mathcal{F}_{t}\right\}\right)$, and $U \in L^{p}\left(\Omega ; W^{1, p}(D)\right)$. Then there exists a unique weak solution $\bar{\pi}=\left(\bar{\Omega}, \overline{\mathcal{F}}, \overline{\mathbb{P}},\left\{\overline{\mathcal{F}}_{t}\right\}, \bar{N}, \bar{u}, \bar{U}\right)$ of the problem (1.1) in the sense of Definition 2.1. Moreover

i) $\mathcal{L}(U)=\mathcal{L}(\bar{U})$ on $W^{1, p}(D)$ with $\bar{U} \in L^{p}\left(\bar{\Omega} ; W^{1, p}(D)\right)$, and

ii) there exists a positive constant $C>0$ such that

$$
\overline{\mathbb{E}}\left[\sup _{0 \leq t \leq T}\|\bar{u}(t)\|_{L^{2}(D)}^{2}+\int_{0}^{T}\|\bar{u}(t)\|_{W_{0}^{1, p}(D)}^{p} \mathrm{~d} t\right] \leq C \overline{\mathbb{E}}\left[\left\|u_{0}\right\|_{L^{2}(D)}^{2}+\|U\|_{W^{1, p}(D)}^{p}\right] .
$$

Remark 2.1. We remark that the assumption A.3 is natural in the context of Lévy noise with the exception of $\lambda^{*} \in(0,1)$, which is necessary to handle the nonlocal nature of the Itô-Lévy formula for the path-wise uniqueness in Subsection 3.5.1; see also [2, Remark 1]. Finally, the assumptions A.1 A.4 collectively ensures existence of a weak solution of the problem (1.1).

\footnotetext{
${ }^{2}$ Here we denote $x \wedge y:=\min \{x, y\}$.
} 
Remark 2.2. In view of $\left[28 \text {, Remark 1.1], } \overline{\mathbb{P}} \text {-a.s., } \bar{u} \in C_{w}\left([0, T] ; L^{2}(D)\right)\right]^{3}$ and hence for all $t \in[0, T], u(t, \cdot)$ is a stochastic process with values in $L^{2}(D)$.

We denote by $\mathcal{U}_{\mathrm{ad}}^{w}\left(u_{0} ; T\right)$ the set of weak admissible solutions to the problem (1.1) in the sense Theorem 2.1. Then, the initial value control problem (1.2) can be re-written as follows.

Definition 2.2. Let the assumptions of Theorem 2.1 hold, and let $\Psi$ be a given Lipschitz continuous function on $L^{2}(D)$ and $u_{\operatorname{tar}} \in L^{p}\left(0, T ; W_{0}^{1, p}(D)\right)$ be a given deterministic target profile. A weak optimal solution of (1.2) is a 7-tuple $\pi^{*}:=\left(\Omega^{*}, \mathcal{F}^{*}, \mathbb{P}^{*},\left\{\mathcal{F}_{t}^{*}\right\}, N^{*}, u^{*}, U^{*}\right) \in$ $\mathcal{U}_{\mathrm{ad}}^{w}\left(u_{0} ; T\right)$ such that

$$
\mathcal{J}\left(\pi^{*}\right)=\inf _{\pi \in \mathcal{U}_{\mathrm{ad}}^{w}\left(u_{0} ; T\right)} \mathcal{J}(\pi):=\Lambda .
$$

The associated control $U^{*}$ in $\pi^{*}$, as in (2.2) is called weak optimal control of the control problem (1.2).

Theorem 2.2. There exists a weak optimal solution $\pi^{*}$ of (1.2) in the sense of Definition 2.2 .

\section{WELLPOSEDNESS OF WEAK SOLUTION}

In this section, we establish wellposedness of a weak solution for (1.1). To do this, we first construct an approximate solution via implicit time discretization scheme, and then derive necessary uniform bounds.

3.1. Implicit Euler scheme. For $N \in \mathbb{N}^{*}$, let $0=t_{0}<t_{1}<\cdots<t_{N}=T$ be a uniform partition of $[0, T]$ with mesh size $\Delta t:=\frac{T}{N}>0$ i.e., $t_{k}=k \Delta t$ for $0 \leq k \leq N$.

For any $u_{0} \in L^{2}(D)$, there exists a sequence $\left\{u_{0, \Delta t}\right\} \in W_{0}^{1, p}(D)$ such that $u_{0, \Delta t} \rightarrow u_{0}$ in $L^{2}(D)$ as $\Delta t \rightarrow 0$. Moreover, the following estimate holds:

$$
\frac{1}{2}\left\|u_{0, \Delta t}\right\|_{L^{2}(D)}^{2}+\Delta t\left\|\nabla u_{0, \Delta t}\right\|_{L^{p}(D)}^{p} \leq \frac{1}{2}\left\|u_{0}\right\|_{L^{2}(D)}^{2} .
$$

For its proof, we refer to see [28, Lemma 30]. Set $\hat{u}_{0}=u_{0, \Delta t}+U$. With this $\hat{u}_{0}$, we introduce the following time discretization:

$$
\hat{u}_{k+1}-\hat{u}_{k}-\Delta t \operatorname{div}_{x}\left(\left|\nabla \hat{u}_{k+1}\right|^{p-2} \nabla \hat{u}_{k+1}+\vec{f}\left(\hat{u}_{k+1}\right)\right)=\int_{|z|>0} \int_{t_{k}}^{t_{k+1}} \eta\left(\hat{u}_{k} ; z\right) \widetilde{N}(\mathrm{~d} z, \mathrm{~d} t) .
$$

Proposition 3.1. Let $\Delta t>0$ be small and $\hat{u}_{0}$ is defined as above. Then, for any $k=$ $0,1,2, \cdots, N-1$, there exists a unique $\mathcal{F}_{t_{k+1}}$-measurable $W_{0}^{1, p}(D)$-valued random variable $\hat{u}_{k+1}$ such that for any $v \in W_{0}^{1, p}(D)$, the following variational formula holds

$$
\begin{aligned}
& \int_{D}\left(\left(\hat{u}_{k+1}-\hat{u}_{k}\right) v+\Delta t\left\{\left|\nabla \hat{u}_{k+1}\right|^{p-2} \nabla \hat{u}_{k+1}+\vec{f}\left(\hat{u}_{k+1}\right)\right\} \cdot \nabla v\right) \mathrm{d} x \\
& =\int_{D} \int_{t_{k}}^{t_{k+1}} \int_{|z|>0} \eta\left(\hat{u}_{k} ; z\right) v \widetilde{N}(\mathrm{~d} z, \mathrm{~d} s) \mathrm{d} x .
\end{aligned}
$$

i.e., $\mathbb{P}$-a.s., $\hat{u}_{k+1}$ is a unique weak solution to the problem (3.2).

Proof. Let $\Delta t>0$ be a fixed small number. Define an operator $\mathcal{A}: W_{0}^{1, p}(D) \rightarrow W^{-1, p^{\prime}}(D)$ via

$$
\langle\mathcal{A} u, v\rangle:=\int_{D}\left(u v+\Delta t\left\{|\nabla u|^{p-2} \nabla u+\vec{f}(u)\right\} \cdot \nabla v\right) \mathrm{d} x, \quad \forall u, v \in W_{0}^{1, p}(D) .
$$

\footnotetext{
${ }^{3}$ For any Banach space $\mathbb{X}, C_{w}([0, T] ; \mathbb{X})$ denotes the Bochner space of weakly continuous functions with values in $\mathbb{X}$.
} 
Then, $\mathcal{A}$ is a coercive pseudo-monotone operator and hence by Brezis' theorem $\mathcal{A}$ is onto $W^{-1, p^{\prime}}(D)$, see [26. Theorem 2.6]. Arguing similarly as in the proof of [28, Lemma 1], we infer that $\mathcal{A}$ is injective and $\mathcal{A}^{-1}: W^{-1, p^{\prime}}(D) \rightarrow W_{0}^{1, p}(D)$ is continuous.

Let $X_{k}:=\hat{u}_{k}+\int_{|z|>0} \int_{t_{k}}^{t_{k+1}} \eta\left(\hat{u}_{k} ; z\right) \widetilde{N}(\mathrm{~d} z, \mathrm{~d} t)$. Then, thanks to the assumption $\mathbf{A} .3$ and Itô-Lévy isometry, we have

$$
\begin{aligned}
\mathbb{E}\left[\left\|X_{k}\right\|_{L^{2}(D)}^{2}\right] & \leq 2 \mathbb{E}\left[\left\|\hat{u}_{k}\right\|_{L^{2}(D)}^{2}\right]+2 \lambda^{*} \Delta t \mathbb{E}\left[\int_{|z|>0}\left\|\hat{u}_{k}\right\|_{L^{2}(D)}^{2}\left(1 \wedge|z|^{2}\right) m(\mathrm{~d} z)\right] \\
& \leq C\left(\Delta t, \lambda, c_{\eta}\right) \mathbb{E}\left[\left\|\hat{u}_{k}\right\|_{L^{2}(D)}^{2}\right] .
\end{aligned}
$$

Therefore for a.s. $\omega \in \Omega, X_{k} \in L^{2}(D)$, and hence $\hat{u}_{k+1}=\mathcal{A}^{-1} X_{k}$. Note that $\hat{u}_{k+1}$ is $\mathcal{F}_{t_{k+1}}$ measurable if we assume that $\hat{u}_{k}$ is $\mathcal{F}_{t_{k}}$-measurable. Thus the assertion follows by induction. This completes the proof.

3.2. $\boldsymbol{A}$-priori estimate. We choose a test function $v=\hat{u}_{k+1}$ in (3.3), and use Young's inequality, A.3 A.4, Itô-Lévy isometry, and the identity $(a-b) a=\frac{1}{2}\left[a^{2}+(a-b)^{2}-b^{2}\right]$ for all $a, b \in \mathbb{R}$ to have, after taking the expectation and recalling $\int_{D} \vec{f}(v) \cdot \nabla v d x=0$ for any $v \in W_{0}^{1, p}(D)$,

$$
\begin{aligned}
& \frac{1}{2}\left\{\mathbb{E}\left[\left\|\hat{u}_{k+1}\right\|_{L^{2}(D)}^{2}\right]+\mathbb{E}\left[\left\|\hat{u}_{k+1}-\hat{u}_{k}\right\|_{L^{2}(D)}^{2}\right]-\mathbb{E}\left[\left\|\hat{u}_{k}\right\|_{L^{2}(D)}^{2}\right]\right\}+\Delta t \mathbb{E}\left[\left\|\nabla \hat{u}_{k+1}\right\|_{L^{p}(D)}^{p}\right] \\
& \leq \frac{1}{4} \mathbb{E}\left[\left\|\hat{u}_{k+1}-\hat{u}_{k}\right\|_{L^{2}(D)}^{2}\right]+C \Delta t \mathbb{E}\left[\left\|\hat{u}_{k}\right\|_{L^{2}(D)}^{2}\right] .
\end{aligned}
$$

An application of discrete Gronwall's lemma then implies

$$
\sup _{0 \leq n \leq N} \mathbb{E}\left[\left\|\hat{u}_{n}\right\|_{L^{2}(D)}^{2}\right]+\sum_{k=0}^{N-1} \mathbb{E}\left[\left\|\hat{u}_{k+1}-\hat{u}_{k}\right\|_{L^{2}(D)}^{2}\right]+\Delta t \sum_{k=0}^{N-1} \mathbb{E}\left[\left\|\nabla \hat{u}_{k+1}\right\|_{L^{p}(D)}^{p}\right] \leq C .
$$

Moreover, we can easily show that $\mathbb{E}\left[\sup _{0 \leq n \leq N}\left\|\hat{u}_{n}\right\|_{L^{2}(D)}^{2}\right] \leq C$.

We would like to define certain processes defined on the whole time interval $[0, T]$ in terms of the discrete solutions $\left\{\hat{u}_{k}\right\}$, and derive a-priori estimate. Like in [28, we introduce the right-continuous step function $u_{\Delta t}(t)$, left-continuous $\left\{\mathcal{F}_{t}\right\}$-adapted step function $\bar{u}_{\Delta t}(t)$, squareintegrable $\left\{\mathcal{F}_{t}\right\}$-martingale $B_{\Delta t}(t)$ and the piecewise affine functions $\tilde{u}_{\Delta t}(t)$ and $\tilde{B}_{\Delta t}(t)$ as

$$
\begin{aligned}
u_{\Delta t}(t): & =\sum_{k=0}^{N-1} \hat{u}_{k+1} \mathbf{1}_{\left[t_{k}, t_{k+1}\right)}(t) ; \quad \bar{u}_{\Delta t}(t)=\sum_{k=0}^{N-1} \hat{u}_{k} \mathbf{1}_{\left(t_{k}, t_{k+1}\right]}(t) \text { with } \bar{u}_{\Delta t}(0)=\hat{u}_{0}, \\
B_{\Delta t}(t): & =\int_{0}^{t} \int_{|z|>0} \eta\left(u_{\Delta t}(s) ; z\right) \widetilde{N}(\mathrm{~d} z, \mathrm{~d} s),
\end{aligned}
$$

and

$$
\begin{aligned}
\tilde{u}_{\Delta t}(t) & :=\sum_{k=0}^{N-1}\left(\frac{\hat{u}_{k+1}-\hat{u}_{k}}{\Delta t}\left(t-t_{k}\right)+\hat{u}_{k}\right) \mathbf{1}_{\left[t_{k}, t_{k+1}\right)}(t) \text { with } \tilde{u}_{\Delta t}(T)=\hat{u}_{N}, \\
\tilde{B}_{\Delta t}(t) & :=\sum_{k=0}^{N-1}\left(\frac{B_{\Delta t}\left(t_{k+1}\right)-B_{\Delta t}\left(t_{k}\right)}{\Delta t}\left(t-t_{k}\right)+B_{\Delta t}\left(t_{k}\right)\right) \mathbf{1}_{\left[t_{k}, t_{k+1}\right)}(t) .
\end{aligned}
$$


A straightforward calculation shows that

$$
\left\{\begin{array}{l}
\sup _{t \in[0, T]} \mathbb{E}\left[\left\|u_{\Delta t}(t)\right\|_{L^{2}(D)}^{2}\right]=\max _{0 \leq k \leq N-1} \mathbb{E}\left[\left\|\hat{u}_{k+1}\right\|_{L^{2}(D)}^{2}\right], \\
\mathbb{E}\left[\sup _{t \in[0, T]}\left\|u_{\Delta t}(t)\right\|_{L^{2}(D)}^{2}\right] \leq \mathbb{E}\left[\max _{0 \leq k \leq N-1}\left\|\hat{u}_{k+1}\right\|_{L^{2}(D)}^{2}\right], \\
\mathbb{E}\left[\left\|u_{\Delta t}-\tilde{u}_{\Delta t}\right\|_{L^{2}\left(D_{T}\right)}^{2}\right] \leq \Delta t \sum_{k=0}^{N-1} \mathbb{E}\left[\left\|\hat{u}_{k+1}-\hat{u}_{k}\right\|_{L^{2}(D)}^{2}\right] .
\end{array}\right.
$$

In view of the above definitions and a-priori estimate (3.4), we arrive at the following lemma.

Lemma 3.2. There exists a constant $C>0$, independent of $\Delta t$, such that

$$
\begin{aligned}
& \sup _{t \in[0, T]} \mathbb{E}\left[\left\|u_{\Delta t}(t)\right\|_{L^{2}(D)}^{2}\right]=\sup _{t \in[0, T]} \mathbb{E}\left[\left\|\tilde{u}_{\Delta t}(t)\right\|_{L^{2}(D)}^{2}\right] \leq C, \\
& \mathbb{E}\left[\sup _{t \in[0, T]}\left\|u_{\Delta t}(t)\right\|_{L^{2}(D)}^{2}\right]=\mathbb{E}\left[\sup _{t \in[0, T]}\left\|\tilde{u}_{\Delta t}(t)\right\|_{L^{2}(D)}^{2}\right] \leq C, \\
& \mathbb{E}\left[\int_{0}^{T} \int_{D}\left|\nabla u_{\Delta t}(t)\right|^{p} \mathrm{~d} x \mathrm{~d} t\right] \leq C ; \quad \mathbb{E}\left[\int_{0}^{T} \int_{D}\left|\nabla \tilde{u}_{\Delta t}(t)\right|^{p} \mathrm{~d} x \mathrm{~d} t\right] \leq C, \\
& \mathbb{E}\left[\left\|u_{\Delta t}-\tilde{u}_{\Delta t}\right\|_{L^{2}\left(D_{T}\right)}^{2}\right] \leq C \Delta t .
\end{aligned}
$$

Thanks to (3.1) and (3.6), one can easily show the following estimate:

$$
\begin{aligned}
\mathbb{E}\left[\left\|\tilde{u}_{\Delta t}\right\|_{L^{p}\left(0, T ; W_{0}^{1, p}(D)\right)}^{p}\right] & \leq C \mathbb{E}\left[\int_{0}^{T}\left\|\nabla u_{\Delta t}(t)\right\|_{L^{p}(D)}^{p} \mathrm{~d} t+\Delta t\left\|\nabla \hat{u}_{0}\right\|_{L^{p}(D)}^{p}\right] \\
& \leq C\left(\left\|u_{0}\right\|_{L^{2}(D)}^{2}+\mathbb{E}\left[\|U\|_{W^{1, p}(D)}^{p}\right]\right),
\end{aligned}
$$

for some constant $C>0$, independent of $\Delta t$.

3.3. Tightness of the sequence $\mathcal{L}\left(\tilde{u}_{\Delta t}\right)$. In this subsection, we will show that the laws of the sequence $\tilde{u}_{\Delta t}$, denoted by $\mathcal{L}\left(\tilde{u}_{\Delta t}\right)$, is tight on some appropriate functional space. To do so, analogous to those considered in [6, 16, 17, we define

$$
\mathcal{Z}:=\mathbb{D}\left([0, T] ; W^{-1, p^{\prime}}(D)\right) \cap \mathbb{D}\left([0, T] ; L_{w}^{2}(D)\right) \cap L_{w}^{2}\left(0, T ; L^{2}(D)\right) \cap L^{2}\left(0, T ; L^{2}(D)\right)
$$

equipped with the topology $\mathcal{T}$, the supremum of the corresponding topologies, where the functional spaces $\mathbb{D}\left([0, T] ; W^{-1, p^{\prime}}(D)\right), L_{w}^{2}\left(0, T ; L^{2}(D)\right)$, and $\mathbb{D}\left([0, T] ; L_{w}^{2}(D)\right)$ endowed with the respective topologies are defined as

1). $\mathbb{D}\left([0, T] ; W^{-1, p^{\prime}}(D)\right):=$ the space of càdlàg functions $u:[0, T] \rightarrow W^{-1, p^{\prime}}(D)$ with the extended Skorokhod topology 4 .

2). $L_{w}^{2}\left(0, T ; L^{2}(D)\right):=$ the space $L^{2}\left(0, T ; L^{2}(D)\right)$ with the weak topology.

$3) . \mathbb{D}\left([0, T] ; L_{w}^{2}(D)\right):=$ the space of all weakly càdlàg functions $u:[0, T] \rightarrow L^{2}(D)$ with the weakest topology such that for all $h \in L^{2}(D)$, the mapping $\mathbb{D}\left([0, T] ; L_{w}^{2}(D)\right) \ni u \mapsto$ $\int_{D} u(\cdot) h \mathrm{~d} x \in \mathbb{D}([0, T] ; \mathbb{R})$ are continuous.

Definition 3.1. (Aldous condition) Let $\left(X_{n}\right)_{n \in \mathbb{N}}$ be a sequence of càdlàg, $\left\{\mathcal{F}_{t}\right\}$-adapted stochastic processes in a Banach space $\mathbb{U}$. We say that $\left(X_{n}\right)_{n \in \mathbb{N}}$ satisfies the Aldous condition if for every $\varepsilon>0$ and $\gamma>0$, there is $\delta>0$ such that for every sequence $\left(\tau_{n}\right)_{n \in \mathbb{N}}$ of $\left\{\mathcal{F}_{t}\right\}$-stopping times with $\tau_{n} \leq T$, one has

$$
\sup _{n \in \mathbb{N}} \sup _{0<\theta \leq \delta} \mathbb{P}\left\{\left\|X_{n}\left(\tau_{n}+\theta\right)-X_{n}\left(\tau_{n}\right)\right\|_{\mathbb{U}} \geq \gamma\right\} \leq \varepsilon .
$$

\footnotetext{
${ }^{4}$ For the Skorokhod topology, we refer to see [1, 27] and references therein.
} 
The following lemma ensures the Aldous condition in a separable Banach space $\mathbb{U}$ for the sequence $\left(X_{n}\right)_{n \in \mathbb{N}} ;$ cf. [19, Lemma 9].

Lemma 3.3. Let $\left(\mathbb{U},\|\cdot\|_{\mathbb{U}}\right)$ be a separable Banach space and let $\left(X_{n}\right)_{n \in \mathbb{N}}$ be a sequence of $\mathbb{U}$-valued random variables. Assume that for every sequence $\left(\tau_{n}\right)$ of $\left\{\mathcal{F}_{t}\right\}$-stopping times with $\tau_{n} \leq T$ and $\theta \geq 0$, the following condition holds

$$
\mathbb{E}\left[\left\|X_{n}\left(\tau_{n}+\theta\right)-X_{n}\left(\tau_{n}\right)\right\|_{\mathbb{U}}^{\alpha}\right] \leq C \theta^{\zeta}
$$

for some $\alpha, \zeta>0$ and some constant $C>0$. Then the sequence $\left(X_{n}\right)_{n \in \mathbb{N}}$ satisfies the Aldous condition.

In view of [18, Lemma 2.5], [19, Theorem 2], see also [6, Lemma 3.3] and [19, Lemma 7], we arrive at the following useful theorem regarding the criterion for the tightness in $\mathcal{Z}$. For its proof, consult [19, Corollary 1].

Theorem 3.4. Let $\left(u_{\Delta t}\right)_{\Delta t>0}$ be a sequence of càdlàg, $W^{-1, p^{\prime}}(D)$-valued stochastic processes such that

i) there exists a constant $C_{1}>0$ such that

$$
\sup _{\Delta t>0} \mathbb{E}\left[\sup _{t \in[0, T]}\left\|u_{\Delta t}(t)\right\|_{L^{2}(D)}\right] \leq C_{1},
$$

ii) there exists a constant $C_{2}>0$ such that

$$
\sup _{\Delta t>0} \mathbb{E}\left[\int_{0}^{T}\left\|u_{\Delta t}(t)\right\|_{W_{0}^{1, p}(D)}^{2} \mathrm{~d} t\right] \leq C_{2},
$$

iii) $\left(u_{\Delta t}\right)_{\Delta t>0}$ satisfies the Aldous condition in $W^{-1, p^{\prime}}(D)$.

Then the sequence $\left(\mathcal{L}\left(u_{\Delta t}\right)\right)_{\Delta t>0}$ is tight on $(\mathcal{Z}, \mathcal{T})$.

With the help of Theorem 3.4, we prove the tightness of the laws of the sequence $\left\{\tilde{u}_{\Delta t}\right\}$ in $(\mathcal{Z}, \mathcal{T})$.

Lemma 3.5. The sequence $\left(\mathcal{L}\left(\tilde{u}_{\Delta t}\right)\right)_{\Delta t>0}$ is tight on $(\mathcal{Z}, \mathcal{T})$.

Proof. Thanks to the a-priori estimates (3.6) and (3.7), we see that assumptions i) and ii) of Theorem 3.4 hold for the sequence $\left(\tilde{u}_{\Delta t}\right)_{\Delta t>0}$. Hence it suffices to prove that the sequence $\left(\tilde{u}_{\Delta t}\right)_{\Delta t>0}$ satisfies the Aldous condition in $W^{-1, p^{\prime}}(D)$. Note that, we can rewrite (3.2) in terms of $u_{\Delta t}, \tilde{u}_{\Delta t}$, and $\tilde{B}_{\Delta t}$ as

$$
\begin{aligned}
\tilde{u}_{\Delta t}(t) & =u_{0, \Delta t}+U+\int_{0}^{t} \operatorname{div}_{x}\left(\left|\nabla u_{\Delta t}(s)\right|^{p-2} \nabla u_{\Delta t}(s)+\vec{f}\left(u_{\Delta t}(s)\right)\right) \mathrm{d} s+\tilde{B}_{\Delta t}(t) \\
& :=\hat{u}_{0}+T_{1}^{\Delta t}(t)+T_{2}^{\Delta t}(t) .
\end{aligned}
$$

First note that, since the term $\hat{u}_{0}$ is independent of time, clearly (3.8) is satisfied for any $\alpha, \zeta$. In view of Lemma 3.3, we need to show that $T_{1}^{\Delta t}(t)$ and $T_{2}^{\Delta t}(t)$ satisfy the inequality (3.8) for a suitable choices of $\alpha, \zeta$. Let $\left(\tau_{m}\right)$ be a sequence of stopping times with $\tau_{m} \leq T$, and $\theta>0$. Then, by using (3.6) we have

$$
\begin{aligned}
& \mathbb{E}\left[\left\|T_{1}^{\Delta t}\left(\tau_{m}+\theta\right)-T_{1}^{\Delta t}\left(\tau_{m}\right)\right\|_{W^{-1, p^{\prime}}(D)}\right] \\
& =\mathbb{E}\left[\left\|\int_{\tau_{m}}^{\tau_{m}+\theta} \operatorname{div}_{x}\left(\left|\nabla u_{\Delta t}(s)\right|^{p-2} \nabla u_{\Delta t}(s)+\vec{f}\left(u_{\Delta t}(s)\right)\right) \mathrm{d} s\right\|_{W^{-1, p^{\prime}}(D)}\right] \\
& \leq \mathbb{E}\left[\int_{\tau_{m}}^{\tau_{m}+\theta}\left\|\left|\nabla u_{\Delta t}(s)\right|^{p-2} \nabla u_{\Delta t}(s)+\vec{f}\left(u_{\Delta t}(s)\right)\right\|_{L^{p^{\prime}}(D)} \mathrm{d} s\right]
\end{aligned}
$$




$$
\begin{aligned}
& \leq C \mathbb{E}\left[\int_{\tau_{m}}^{\tau_{m}+\theta}\left(\left\|\nabla u_{\Delta t}(s)\right\|_{L^{p}(D)}^{p-1}+\left\|u_{\Delta t}(s)\right\|_{L^{p^{\prime}}(D)}\right) \mathrm{d} s\right] \\
& \leq C \theta^{\frac{1}{p}} \mathbb{E}\left[\int_{0}^{T}\left(\left\|\nabla u_{\Delta t}(s)\right\|_{L^{p}(D)}^{p}+\left\|u_{\Delta t}(s)\right\|_{L^{p^{\prime}(D)}}^{p^{\prime}}\right) \mathrm{d} s\right] \leq C \theta^{\frac{1}{2}} .
\end{aligned}
$$

Thus $T_{1}^{\Delta t}(t)$ satisfies (3.8) with $\alpha=1$ and $\zeta=\frac{1}{2}$. Again, thanks to Itô-Lévy isometry, the assumptions A.3.4. and (3.6), and since $W_{0}^{1, p}(D) \hookrightarrow L^{2}(D) \hookrightarrow W^{-1, p^{\prime}}(D)$, we see that

$$
\begin{aligned}
& \mathbb{E}\left[\left\|T_{2}^{\Delta t}\left(\tau_{m}+\theta\right)-T_{2}^{\Delta t}\left(\tau_{m}\right)\right\|_{W^{-1, p^{\prime}}(D)}^{2}\right] \\
& \leq \mathbb{E}\left[\left\|\int_{\tau_{m}}^{\tau_{m}+\theta} \int_{|z|>0} \eta\left(\bar{u}_{\Delta t}(s) ; z\right) \tilde{N}(\mathrm{~d} z, \mathrm{~d} s)\right\|_{W^{-1, p^{\prime}}(D)}^{2}\right] \\
& \leq C \mathbb{E}\left[\left\|\int_{\tau_{m}}^{\tau_{m}+\theta} \int_{|z|>0} \eta\left(\bar{u}_{\Delta t}(s) ; z\right) \tilde{N}(\mathrm{~d} z, \mathrm{~d} s)\right\|_{L^{2}(D)}^{2}\right] \\
& \leq C \mathbb{E}\left[\int_{\tau_{m}}^{\tau_{m}+\theta} \int_{|z|>0}\left\|\eta\left(\bar{u}_{\Delta t}(s) ; z\right)\right\|_{L^{2}(D)}^{2} m(\mathrm{~d} z) \mathrm{d} s\right] \\
& \leq C \mathbb{E}\left[\int_{\tau_{m}}^{\tau_{m}+\theta} \int_{|z|>0}\left\|\bar{u}_{\Delta t}(s)\right\|_{L^{2}(D)}^{2}\left(1 \wedge|z|^{2}\right) m(\mathrm{~d} z) \mathrm{d} s\right] \\
& \leq C c_{\eta} \mathbb{E}\left[\int_{\tau_{m}}^{\tau_{m}+\theta}\left\|\bar{u}_{\Delta t}(s)\right\|_{L^{2}(D)}^{2} \mathrm{~d} s\right] \leq C \theta \mathbb{E}\left[\sup _{s \in[0, T]}\left\|u_{\Delta t}(s)\right\|_{L^{2}(D)}^{2}\right] \leq C \theta .
\end{aligned}
$$

Hence $T_{2}^{\Delta t}(t)$ satisfies (3.8) with $\alpha=2$ and $\zeta=1$. This completes the proof.

3.4. Construction of a martingale solution. Construction of a martingale solution is based on Skorokhod Theorem [14] for a non metric space. Note that $\mathcal{Z}$ is a locally convex topological space and there exist a sequence of continuous functions $f_{m}: \mathcal{Z} \rightarrow \mathbb{R}$ that separates the points of $\mathcal{Z}$ which generates the Borel $\sigma$-algebra; cf. [19, Remark 2]. Let $\overline{\mathbb{N}}$ denotes the set of all extended natural numbers i.e., $\overline{\mathbb{N}}:=\mathbb{N} \cup\{\infty\}$. For any measurable space $(S, \mathcal{B}(S))$, we denote by $M_{\overline{\mathbb{N}}}(S)$ the set of all $\overline{\mathbb{N}}$-valued measures on $(S, \mathcal{B}(S))$ endowed with the $\sigma$-field $\mathcal{M}_{\overline{\mathbb{N}}}(S)$ generated by the projection maps $i_{B}: M_{\overline{\mathbb{N}}}(S) \ni \mu \mapsto \mu(B) \in \overline{\mathbb{N}}$ for all $B \in \mathcal{B}(S)$. Define $N_{\Delta t}(\mathrm{~d} z, \mathrm{~d} t)=N(\mathrm{~d} z, \mathrm{~d} t)$ for all $\Delta t>0$. For the basic properties of the stochastic integral with respect to compensated Poisson random measure $\widetilde{N}$, we refer to see [4, 13] and [25]. Since $M_{\overline{\mathbb{N}}}(\mathbb{R} \times[0, T])$ is a separable metric space, by [24, Theorem 3.2], the laws of the family $\left\{N_{\Delta t}(\mathrm{~d} z, \mathrm{~d} t)\right\}$ is tight on $M_{\overline{\mathbb{N}}}(\mathbb{R} \times[0, T])$.

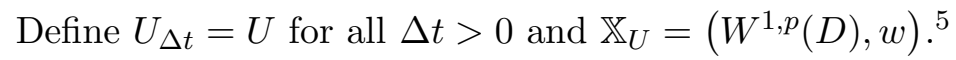

Lemma 3.6. The set $\left\{\mathcal{L}\left(U_{\Delta t}\right): \Delta t>0\right\}$ is tight in $\mathbb{X}_{U}$.

Proof. Note that $\sup _{\Delta t>0} \mathbb{E}\left[\left\|U_{\Delta t}\right\|_{W^{1, p}(D)}^{p}\right]=\mathbb{E}\left[\|U\|_{W^{1, p}(D)}^{p}\right]<+\infty$. Now for any $R>0$, the set

$$
B_{R}:=\left\{U \in W^{1, p}(D):\|U\|_{W^{1, p}(D)} \leq R\right\}
$$

is relatively compact in $\mathbb{X}_{U}$ and

$$
\mathbb{P}\left(\left\|U_{\Delta t}\right\|_{W^{1, p}(D)} \geq R\right) \leq \frac{1}{R^{p}} \mathbb{E}\left[\left\|U_{\Delta t}\right\|_{W^{1, p}(D)}^{p}\right] \leq \frac{C}{R^{p}},
$$

which yields the proof.

\footnotetext{
${ }^{5}$ We denote by $(\mathbb{Y}, w)$ the topological space $\mathbb{Y}$ equipped with the weak topology.
} 
By Lemma 3.5, the set of measures $\left(\mathcal{L}\left(\tilde{u}_{\Delta t}\right)\right)_{\Delta t>0}$ is tight on $(\mathcal{Z}, \mathcal{T})$. Hence, in view of Lemma 3.6, the set $\left\{\mathcal{L}\left(\tilde{u}_{\Delta t}, U_{\Delta t}, N_{\Delta t}\right): \Delta t>0\right\}$ is tight on $\mathcal{X}:=\mathcal{Z} \times \mathbb{X}_{U} \times M_{\overline{\mathbb{N}}}(\mathbb{R} \times[0, T])$. Note that the space $\mathcal{X}$ is non-metric space, and hence our compactness argument is based on the JakubowskiSkorokhod representation theorem. Moreover, by using [19, Corollary 2], see also [5, Theorem $D 1$ ], we arrive at the following result.

Proposition 3.7. There exist a subsequence of $\{\Delta t\}$, still we denote it by same $\{\Delta t\}$, a probability space $(\bar{\Omega}, \overline{\mathcal{F}}, \overline{\mathbb{P}})$ and, on this space $\mathcal{X}$-valued random variables $\left(u_{*}, U_{*}, N_{*}\right)$ and $\left(u_{\Delta t}^{*}, U_{\Delta t}^{*}, N_{\Delta t}^{*}\right)$ such that

a). $\mathcal{L}\left(u_{\Delta t}^{*}, U_{\Delta t}^{*}, N_{\Delta t}^{*}\right)=\mathcal{L}\left(\tilde{u}_{\Delta t}, U_{\Delta t}, N_{\Delta t}\right)$ for all $\Delta t>0$,

b). $\left(u_{\Delta t}^{*}, U_{\Delta t}^{*}, N_{\Delta t}^{*}\right) \rightarrow\left(u_{*}, U_{*}, N_{*}\right)$ in $\mathcal{X} \quad \overline{\mathbb{P}}$-a.s. as $\Delta t \rightarrow 0$,

c). $N_{\Delta t}^{*}(\bar{\omega})=N_{*}(\bar{\omega})$ for all $\bar{\omega} \in \bar{\Omega}$.

Moreover, by [29, Theorem 1.10.4 \& Addendum 1.10.5], there exist a sequence of perfect functions $\phi_{\Delta t}: \bar{\Omega} \rightarrow \Omega$ such that

$$
u_{\Delta t}^{*}=\tilde{u}_{\Delta t} \circ \phi_{\Delta t}, \quad U_{\Delta t}^{*}=U_{\Delta t} \circ \phi_{\Delta t}, \quad \mathbb{P}=\overline{\mathbb{P}} \circ \phi_{\Delta t}^{-1} .
$$

Let $\overline{\mathbb{F}}:=\left(\overline{\mathcal{F}}_{t}\right)_{t \in[0, T]}$ be the filtration defined by

$$
\overline{\mathcal{F}}_{t}:=\sigma\left\{\left(u_{\Delta t}^{*}(s), N_{\Delta t}^{*}(s), u_{*}(s)\right): 0 \leq s \leq t\right\}, \quad t \in[0, T] .
$$

Note that since $N_{\Delta t}^{*}(\bar{\omega})=N_{*}(\bar{\omega})$ for all $\bar{\omega} \in \bar{\Omega}$, the filtration obtained by replacing $N_{\Delta t}^{*}$ by $N_{*}$ in (3.11) is equal to $\overline{\mathbb{F}}$. Moreover, $N_{\Delta t}^{*}, N_{*}$ are time homogeneous Poisson random measures on $\mathbb{R}$ over the stochastic basis $(\bar{\Omega}, \overline{\mathcal{F}}, \overline{\mathbb{P}}, \mathbb{F})$ with intensity measure $m(\mathrm{~d} z)$; cf. [5, Section 9].

Let us define

$$
\begin{aligned}
v_{k} & =\hat{u}_{k} \circ \phi_{\Delta t}, \quad k=0,1, \cdots, N \\
v_{\Delta t}(t) & =\sum_{k=0}^{N-1} v_{k+1} \mathbf{1}_{\left[t_{k}, t_{k+1}\right)}(t) \quad t \in[0, T] \\
\bar{v}_{\Delta t}(t) & =\sum_{k=0}^{N-1} v_{k} \mathbf{1}_{\left(t_{k}, t_{k+1}\right]}(t) \quad t \in(0, T] \text { with } \bar{v}_{\Delta t}(0)=u_{0, \Delta t}+U_{\Delta t}^{*}, \\
B_{\Delta t}^{*}(t) & =\int_{0}^{t} \int_{|z|>0} \eta\left(\bar{v}_{\Delta t}(s) ; z\right) \tilde{N}_{\Delta t}^{*}(\mathrm{~d} z, \mathrm{~d} s) .
\end{aligned}
$$

Note that, thanks to (3.10), (3.12) and (3.2), we have, for any $k=0,1, \cdots, N-1$ and $\overline{\mathbb{P}}$ a.s.,

$$
\begin{aligned}
& v_{k+1}-v_{k}-\Delta t \operatorname{div}_{x}\left(\left|\nabla v_{k+1}\right|^{p-2} \nabla v_{k+1}+\vec{f}\left(v_{k+1}\right)\right)=\int_{|z|>0} \int_{t_{k}}^{t_{k+1}} \eta\left(v_{k} ; z\right) \widetilde{N}_{\Delta t}^{*}(\mathrm{~d} z, \mathrm{~d} t), \\
& u_{\Delta t}^{*}(t)=\sum_{k=0}^{N-1}\left(\frac{v_{k+1}-v_{k}}{\Delta t}\left(t-t_{k}\right)+v_{k}\right) \mathbf{1}_{\left[t_{k}, t_{k+1}\right)}(t), \quad t \in[0, T) \text { with } u_{\Delta t}^{*}(T)=v_{N} .
\end{aligned}
$$

Moreover the estimate (3.4) remains valid for $v_{k}: k=0,1, \cdots, N$. Furthermore, thanks to (3.6)-(3.7), and Proposition 3.7, there hold

$$
\begin{aligned}
& \overline{\mathbb{E}}\left[\sup _{t \in[0, T]}\left\|u_{\Delta t}^{*}(t)\right\|_{L^{2}(D)}^{2}\right]=\overline{\mathbb{E}}\left[\sup _{t \in[0, T]}\left\|v_{\Delta t}(t)\right\|_{L^{2}(D)}^{2}\right] \leq C\left(\left\|u_{0}\right\|_{L^{2}(D)}^{2}+\overline{\mathbb{E}}\left[\left\|U_{*}\right\|_{L^{2}(D)}^{2}\right]\right), \\
& \overline{\mathbb{E}}\left[\left\|u_{\Delta t}^{*}\right\|_{L^{p}\left(0, T ; W_{0}^{1, p}(D)\right)}\right] \leq C \overline{\mathbb{E}}\left[\left\|u_{0}\right\|_{L^{2}(D)}^{2}+\left\|U_{*}\right\|_{W^{1, p}(D)}^{p}\right] .
\end{aligned}
$$

Lemma 3.8. We have the following:

i) $u_{\Delta t}^{*} \rightarrow u_{*}$ in $L^{q}\left(\bar{\Omega} ; L^{2}\left(0, T ; L^{2}(D)\right)\right)$ for all $1 \leq q<p$. 
ii) $v_{\Delta t} \rightarrow u_{*}$ in $L^{2}\left(\bar{\Omega} ; L^{2}\left(0, T ; L^{2}(D)\right)\right)$.

iii) $u_{\Delta t}^{*} \stackrel{*}{\rightarrow} u_{*}$ in $L_{w}^{2}\left(\bar{\Omega} ; L^{\infty}\left(0, T ; L^{2}(D)\right)\right)$.

Proof. We use the estimates (3.15)-(3.16) to prove the lemma.

Proof of i). In view of (3.14), (3.16) and the definition of $v_{\Delta t}$ in (3.12), we see that the sequence $\left\{u_{\Delta t}^{*}\right\}$ is uniformly bounded in $L^{p}\left(\bar{\Omega} ; L^{2}\left(0, T ; L^{2}(D)\right)\right)$ and therefore equi-integrable in $L^{q}\left(\bar{\Omega} ; L^{2}\left(0, T ; L^{2}(D)\right)\right)$ for all $1 \leq q<p$. Since $\overline{\mathbb{P}}$-a.s., $u_{\Delta t}^{*} \rightarrow u_{*}$ in $\mathcal{Z}$ (in particular, $u_{\Delta t}^{*} \rightarrow u_{*}$ in $\left.L^{2}\left(0, T ; L^{2}(D)\right)\right)$, by Vitali convergence theorem we conclude that i) holds as well.

Proof of ii). A straightforward calculation reveals that

$$
\overline{\mathbb{E}}\left[\int_{0}^{T}\left\|u_{\Delta t}^{*}(t)-v_{\Delta t}\right\|_{L^{2}(D)}^{2} \mathrm{~d} t\right] \leq C \Delta t
$$

Thanks to i), we see that $u_{\Delta t}^{*} \rightarrow u_{*}$ in $L^{2}\left(\bar{\Omega} ; L^{2}\left(0, T ; L^{2}(D)\right)\right)$ and hence ii) follows from (3.17).

Proof of iii). Note that, by (3.15), the sequence $\left\{u_{\Delta t}^{*}\right\}$ is uniformly bounded in $L^{2}\left(\bar{\Omega} ; L^{\infty}\left(0, T ; L^{2}(D)\right)\right)$. Since $L^{2}\left(\bar{\Omega} ; L^{\infty}\left(0, T ; L^{2}(D)\right)\right)$ is isomorphic to the space $\left(L^{2}\left(\bar{\Omega} ; L^{1}\left(0, T ; L^{2}(D)\right)\right)\right)^{*}$, by Banach Alaoglu theorem there exist a subsequence, still denoted by $\left\{u_{\Delta t}^{*}\right\}$, and $Y \in L^{2}\left(\bar{\Omega} ; L^{\infty}\left(0, T ; L^{2}(D)\right)\right)$ such that for all $\psi \in L^{2}\left(\bar{\Omega} ; L^{1}\left(0, T ; L^{2}(D)\right)\right)$

$$
\overline{\mathbb{E}}\left[\int_{0}^{T} \int_{D} u_{\Delta t}^{*}(t, x) \psi(t, x) \mathrm{d} x \mathrm{~d} t\right] \rightarrow \overline{\mathbb{E}}\left[\int_{0}^{T} \int_{D} Y(t, x) \psi(t, x) \mathrm{d} x \mathrm{~d} t\right] .
$$

Observe that, thanks to i), $u_{\Delta t}^{*} \rightarrow u_{*}$ in $L^{2}\left(\bar{\Omega} ; L^{2}\left(0, T ; L^{2}(D)\right)\right)$. Since $L^{2}\left(\bar{\Omega} ; L^{2}\left(0, T ; L^{2}(D)\right)\right)$ is a dense subspace of $L^{2}\left(\bar{\Omega} ; L^{1}\left(0, T ; L^{2}(D)\right)\right)$, we conclude that

$$
Y=u_{*} \quad \text { and } \quad u_{*} \in L^{2}\left(\bar{\Omega} ; L^{\infty}\left(0, T ; L^{2}(D)\right)\right) .
$$

This completes the proof.

Lemma 3.9. For all $\phi \in W_{0}^{1, p}(D)$, the following holds

$$
\lim _{\Delta t \rightarrow 0} \overline{\mathbb{E}}\left[\int_{0}^{T}\left|\int_{0}^{t} \int_{|z|>0}\left\langle\eta\left(\bar{v}_{\Delta t}(s) ; z\right)-\eta\left(u_{*}(s-) ; z\right), \phi\right\rangle \widetilde{N}_{*}(\mathrm{~d} z, \mathrm{~d} s)\right|^{2} \mathrm{~d} t\right]=0 .
$$

Proof. By using A.3 A.4, we observe that for any $\phi \in L^{2}(D)$,

$$
\begin{aligned}
& \overline{\mathbb{E}}\left[\int_{0}^{t} \int_{|z|>0}\left|\left(\eta\left(\bar{v}_{\Delta t}(s) ; z\right)-\eta\left(u_{*}(s-) ; z\right), \phi\right)_{L^{2}(D)}\right|^{2} m(\mathrm{~d} z) \mathrm{d} s\right] \\
& \leq\|\phi\|_{L^{2}(D)}^{2} \overline{\mathbb{E}}\left[\int_{0}^{t} \int_{|z|>0}\left\|\eta\left(\bar{v}_{\Delta t}(s) ; z\right)-\eta\left(u_{*}(s-) ; z\right)\right\|_{L^{2}(D)}^{2} m(\mathrm{~d} z) \mathrm{d} s\right] \\
& \leq C\|\phi\|_{L^{2}(D)}^{2} \overline{\mathbb{E}}\left[\int_{0}^{T}\left\|\bar{v}_{\Delta t}(s)-u_{*}(s-)\right\|_{L^{2}(D)}^{2} \mathrm{~d} s\right] .
\end{aligned}
$$

Note that by i) of Lemma 3.8, $u_{\Delta t}^{*} \rightarrow u_{*}$ in $L^{2}\left(\bar{\Omega}, L^{2}\left(0, T ; L^{2}(D)\right)\right)$, and the same holds for $\bar{v}_{\Delta t}$. Hence

$$
\lim _{\Delta t \rightarrow 0} \overline{\mathbb{E}}\left[\int_{0}^{t} \int_{|z|>0}\left|\left(\eta\left(\bar{v}_{\Delta t}(s) ; z\right)-\eta\left(u_{*}(s-) ; z\right), \phi\right)_{L^{2}(D)}\right|^{2} m(\mathrm{~d} z) \mathrm{d} s\right]=0 .
$$

Moreover, by the assumptions A.3 A.4 and (3.15) along with the fact that $u_{*} \in L^{2}\left(\bar{\Omega} ; L^{\infty}\left(0, T ; L^{2}(D)\right)\right)$, we have

$$
\overline{\mathbb{E}}\left[\int_{0}^{t} \int_{|z|>0}\left|\left(\eta\left(\bar{v}_{\Delta t}(s) ; z\right)-\eta\left(u_{*}(s-) ; z\right), \phi\right)_{L^{2}(D)}\right|^{2} m(\mathrm{~d} z) \mathrm{d} s\right] \leq C
$$


for some constant $C>0$. Furthermore, by using the properties of the stochastic integral with respect to the compensated Poisson random measure and the fact that $N_{\Delta t}^{*}=N_{*}$, we have

$$
\begin{aligned}
& \overline{\mathbb{E}}\left[\left|\int_{0}^{t} \int_{|z|>0}\left(\eta\left(\bar{v}_{\Delta t}(s) ; z\right)-\eta\left(u_{*}(s-) ; z\right), \phi\right)_{L^{2}(D)} \widetilde{N}_{*}(\mathrm{~d} z, \mathrm{~d} s)\right|^{2}\right] \\
& =\overline{\mathbb{E}}\left[\int_{0}^{t} \int_{|z|>0}\left|\left(\eta\left(\bar{v}_{\Delta t}(s) ; z\right)-\eta\left(u_{*}(s-) ; z\right), \phi\right)_{L^{2}(D)}\right|^{2} m(\mathrm{~d} z) \mathrm{d} s\right] .
\end{aligned}
$$

Therefore by (3.19) and (3.20), we have for all $\phi \in L^{2}(D)$

$$
\begin{aligned}
& \lim _{\Delta t \rightarrow 0} \overline{\mathbb{E}}\left[\left|\int_{0}^{t} \int_{|z|>0}\left(\eta\left(\bar{v}_{\Delta t}(s) ; z\right)-\eta\left(u_{*}(s-) ; z\right), \phi\right)_{L^{2}(D)} \widetilde{N}_{*}(\mathrm{~d} z, \mathrm{~d} s)\right|^{2}\right]=0 \\
& \text { and, } \quad \overline{\mathbb{E}}\left[\left|\int_{0}^{t} \int_{|z|>0}\left(\eta\left(\bar{v}_{\Delta t}(s) ; z\right)-\eta\left(u_{*}(s-) ; z\right), \phi\right)_{L^{2}(D)} \widetilde{N}_{*}(\mathrm{~d} z, \mathrm{~d} s)\right|^{2}\right] \leq C .
\end{aligned}
$$

Thus, one can use dominated convergence theorem to conclude

$$
\lim _{\Delta t \rightarrow 0} \overline{\mathbb{E}}\left[\int_{0}^{T}\left|\int_{0}^{t} \int_{|z|>0}\left(\eta\left(\bar{v}_{\Delta t}(s) ; z\right)-\eta\left(u_{*}(s-) ; z\right), \phi\right)_{L^{2}(D)} \widetilde{N}_{*}(\mathrm{~d} z, \mathrm{~d} s)\right|^{2} \mathrm{~d} t\right]=0
$$

for any $\phi \in L^{2}(D)$. Since $W_{0}^{1, p}(D) \subset L^{2}(D)$, (3.18) holds true for all $\phi \in W_{0}^{1, p}(D)$. This finishes the proof.

Define the piecewise affine function

$$
b_{\Delta t}^{*}(t):=\sum_{k=0}^{N-1}\left(\frac{B_{\Delta t}^{*}\left(t_{k+1}\right)-B_{\Delta t}^{*}\left(t_{k}\right)}{\Delta t}\left(t-t_{k}\right)+B_{\Delta t}^{*}\left(t_{k}\right)\right) \mathbf{1}_{\left[t_{k}, t_{k+1}\right)}(t), \quad t \in[0, T] .
$$

Lemma 3.10. We have

$$
\overline{\mathbb{E}}\left[\int_{0}^{T}\left\|B_{\Delta t}^{*}(t)-b_{\Delta t}^{*}(t)\right\|_{L^{2}(D)}^{2} \mathrm{~d} t\right] \leq C \Delta t
$$

Proof. Note that for any $t \in\left[t_{k}, t_{k+1}\right)$, we have $\overline{\mathbb{P}}$-a.s.,

$$
\begin{aligned}
& \left\|B_{\Delta t}^{*}(t)-b_{\Delta t}^{*}(t)\right\|_{L^{2}(D)}^{2} \\
& =\left\|\int_{t_{k}}^{t} \int_{|z|>0} \eta\left(\bar{v}_{\Delta t}(s ; z)\right) \widetilde{N}_{*}(\mathrm{~d} z, \mathrm{~d} s)-\frac{t-t_{k}}{\Delta t} \int_{t_{k}}^{t_{k+1}} \int_{|z|>0} \eta\left(\bar{v}_{\Delta t}(s ; z)\right) \widetilde{N}_{*}(\mathrm{~d} z, \mathrm{~d} s)\right\|_{L^{2}(D)}^{2} \\
& \leq 2\left\|\int_{t_{k}}^{t_{k+1}} \int_{|z|>0} \eta\left(\bar{v}_{\Delta t}(s) ; z\right) \widetilde{N}_{*}(\mathrm{~d} z, \mathrm{~d} s)\right\|_{L^{2}(D)}^{2} .
\end{aligned}
$$

Thanks to Itô-Lévy isometry, the assumption \$.3, and the estimate (3.15) along with (3.21), we obtain

$$
\begin{aligned}
& \overline{\mathbb{E}}\left[\int_{0}^{T}\left\|B_{\Delta t}^{*}(t)-b_{\Delta t}^{*}(t)\right\|_{L^{2}(D)}^{2} \mathrm{~d} t\right] \\
& =\sum_{k=0}^{N-1} \overline{\mathbb{E}}\left[\int_{t_{k}}^{t_{k+1}}\left\|B_{\Delta t}^{*}(t)-b_{\Delta t}^{*}(t)\right\|_{L^{2}(D)}^{2} \mathrm{~d} t\right] \\
& \leq 2 \sum_{k=0}^{N-1} \int_{t_{k}}^{t_{k+1}} \overline{\mathbb{E}}\left[\left\|\int_{t_{k}}^{t_{k+1}} \int_{|z|>0} \eta\left(\bar{v}_{\Delta t}(s) ; z\right) \widetilde{N}_{*}(\mathrm{~d} z, \mathrm{~d} s)\right\|_{L^{2}(D)}^{2}\right] \mathrm{d} t \\
& \leq 2 \sum_{k=0}^{N-1} \int_{t_{k}}^{t_{k+1}} \overline{\mathbb{E}}\left[\int_{t_{k}}^{t_{k+1}} \int_{|z|>0}\left\|\bar{v}_{\Delta t}(s)\right\|_{L^{2}(D)}^{2}\left(1 \wedge|z|^{2}\right) m(\mathrm{~d} z) \mathrm{d} s\right] \mathrm{d} t
\end{aligned}
$$




$$
\leq C \Delta t \overline{\mathbb{E}}\left[\int_{0}^{T}\left\|\bar{v}_{\Delta t}(s)\right\|_{L^{2}(D)}^{2} \mathrm{~d} s\right] \leq C \Delta t .
$$

This completes the proof.

Lemma 3.11. The following holds: for all $\phi \in W_{0}^{1, p}(D)$

$$
\begin{aligned}
& \lim _{\Delta t \rightarrow 0} \overline{\mathbb{E}}\left[\left|\left(u_{\Delta t}^{*}(0)-u_{*}(0), \phi\right)_{L^{2}(D)}\right|\right]=0, \\
& \lim _{\Delta t \rightarrow 0} \overline{\mathbb{E}}\left[\int_{0}^{T}\left|\left\langle b_{\Delta t}^{*}(t), \phi\right\rangle-\left\langle\int_{0}^{t} \int_{|z|>0} \eta\left(u_{*}(s-, \cdot) ; z\right) \widetilde{N}_{*}(\mathrm{~d} z, \mathrm{~d} s), \phi\right\rangle\right| \mathrm{d} t\right]=0, \\
& \lim _{\Delta t \rightarrow 0} \overline{\mathbb{E}}\left[\int_{0}^{T}\left|\int_{0}^{t}\left\langle\operatorname{div}_{x}\left(\vec{f}\left(\bar{v}_{\Delta t}(s)\right)-\vec{f}\left(u_{*}(s)\right)\right), \phi\right\rangle \mathrm{d} s\right| \mathrm{d} t\right]=0,
\end{aligned}
$$

and there exists $G \in L^{p^{\prime}}\left(\bar{\Omega} \times D_{T}\right)^{d}$ such that

$$
\lim _{\Delta t \rightarrow 0} \overline{\mathbb{E}}\left[\int_{0}^{T}\left|\int_{0}^{t}\left\langle\operatorname{div}_{x}\left(\left|\nabla v_{\Delta t}(s)\right|^{p-2} \nabla v_{\Delta t}(s)-G(s)\right), \phi\right\rangle \mathrm{d} s\right| \mathrm{d} t\right]=0 .
$$

Proof. We prove (3.22)-(3.25) step by step.

Proof of (3.22): Note that $\overline{\mathbb{P}}$-a.s., $u_{\Delta t}^{*} \rightarrow u_{*}$ in $\mathbb{D}\left([0, T] ; L_{w}^{2}(D)\right)$ and $u_{*}$ is right continuous at $t=0$. Thus, for any $\phi \in W_{0}^{1, p}(D)$ there holds $\overline{\mathbb{P}}_{\text {-a.s. }},\left(u_{\Delta t}^{*}(0), \phi\right)_{L^{2}(D)} \rightarrow\left(u_{*}(0), \phi\right)_{L^{2}(D)}$. Therefore, one can use (3.15) and Vitali theorem to conclude (3.22).

Proof of (3.23) : Notice that, for any $\phi \in W_{0}^{1, p}(D)$

$$
\begin{aligned}
& \left\langle b_{\Delta t}^{*}(t), \phi\right\rangle-\left\langle\int_{0}^{t} \int_{|z|>0} \eta\left(u_{*}(s-, \cdot) ; z\right) \widetilde{N}_{*}(\mathrm{~d} z, \mathrm{~d} s), \phi\right\rangle \\
& =\left\langle b_{\Delta t}^{*}(t)-B_{\Delta t}^{*}(t)+B_{\Delta t}^{*}(t)-\int_{0}^{t} \int_{|z|>0} \eta\left(u_{*}(s-, \cdot) ; z\right) \widetilde{N}_{*}(\mathrm{~d} z, \mathrm{~d} s), \phi\right\rangle \\
& \leq\|\phi\|_{W_{0}^{1, p}(D)}\left\|b_{\Delta t}^{*}(t)-B_{\Delta t}^{*}(t)\right\|_{W^{-1, p}(D)}+\left|\left\langle B_{\Delta t}^{*}(t)-\int_{0}^{t} \int_{|z|>0} \eta\left(u_{*}(s-, \cdot) ; z\right) \widetilde{N}_{*}(\mathrm{~d} z, \mathrm{~d} s), \phi\right\rangle\right| \\
& \leq C\|\phi\|_{W_{0}^{1, p}(D)}\left\|b_{\Delta t}^{*}(t)-B_{\Delta t}^{*}(t)\right\|_{L^{2}(D)} \\
& \quad+\left|\int_{0}^{t} \int_{|z|>0}\left\langle\eta\left(\bar{v}_{\Delta t}(s, \cdot) ; z\right)-\eta\left(u_{*}(s-, \cdot) ; z\right), \phi\right\rangle \widetilde{N}_{*}(\mathrm{~d} z, \mathrm{~d} s)\right| .
\end{aligned}
$$

One can use Lemmas 3.9 and 3.10 to arrive at (3.23).

Proof of (3.24) : Since $\vec{f}$ is Lipschitz continuous, we have

$$
\overline{\mathbb{E}}\left[\int_{0}^{T}\left|\int_{0}^{t}\left\langle\operatorname{div}_{x}\left(\vec{f}\left(\bar{v}_{\Delta t}(s)\right)-\vec{f}\left(u_{*}(s)\right)\right), \phi\right\rangle \mathrm{d} s\right| \mathrm{d} t\right] \leq C\|\phi\|_{W_{0}^{1, p}(D)}\left\|\bar{v}_{\Delta t}-u_{*}\right\|_{L^{2}\left(\bar{\Omega} \times D_{T}\right)},
$$

and hence (3.24) holds by recalling that $\bar{v}_{\Delta t} \rightarrow u_{*}$ in $L^{2}\left(\bar{\Omega} \times D_{T}\right)$.

Proof of (3.25): Thanks to ii) of Lemma 3.8 and the estimate (3.16), there exists a not relabeled subsequence of $\left\{v_{\Delta t}\right\}$ such that $\nabla v_{\Delta t} \rightarrow \nabla u_{*}$ in $L^{p}\left(\bar{\Omega} \times D_{T}\right)^{d}$ for $\Delta t \rightarrow 0$. Moreover, since $\left.\left.|| \nabla v_{\Delta t}\right|^{p-2} \nabla v_{\Delta t}\right|^{p^{\prime}}=\left|\nabla v_{\Delta t}\right|^{p}$, there exists $G \in L^{p^{\prime}}\left(\bar{\Omega} \times D_{T}\right)^{d}$ such that $\left|\nabla v_{\Delta t}\right|^{p-2} \nabla v_{\Delta t} \rightarrow G$ in $L^{p^{\prime}}\left(\bar{\Omega} \times D_{T}\right)^{d}$ for the same subsequence and $\Delta t \rightarrow 0$. Thus, it is easy to conclude that for any $\phi \in W_{0}^{1, p}(D)$

$$
\lim _{\Delta t \rightarrow 0} \overline{\mathbb{E}}\left[\int_{0}^{T}\left|\int_{0}^{t}\left\langle\operatorname{div}_{x}\left(\left|\nabla v_{\Delta t}\right|^{p-2} \nabla v_{\Delta t}(s)-G(s)\right), \phi\right\rangle \mathrm{d} s\right| \mathrm{d} t\right]=0,
$$

i.e., (3.25) holds true. This completes the proof. 
3.5. Proof of Theorem 2.1. In this subsection, we use Lemmas 3.8 and 3.11 to prove existence of a weak solution of (1.1) in the sense of Definition 2.1 in three steps. Moreover, we show pathwise uniqueness of weak solutions of the problem (1.1) with respect to the same stochastic basis and a given control.

Step i): We define the functionals for all $\phi \in W_{0}^{1, p}(D)$,

$$
\begin{aligned}
& \mathcal{K}_{\Delta t}\left(\tilde{u}_{\Delta t}, U, \tilde{N} ; \phi\right)=\left(\hat{u}_{0}, \phi\right)_{L^{2}(D)}+\int_{0}^{t}\left\langle\operatorname{div}_{x}\left(\left|\nabla u_{\Delta t}\right|^{p-2} \nabla u_{\Delta t}(s)\right), \phi\right\rangle \mathrm{d} s \\
&+\int_{0}^{t}\left\langle\operatorname{div}_{x} \vec{f}\left(u_{\Delta t}(s)\right), \phi\right\rangle \mathrm{d} s+\left\langle\tilde{B}_{\Delta t}(t), \phi\right\rangle, \\
& \mathcal{K}_{\Delta t}^{*}\left(u_{\Delta t}^{*}, U_{\Delta t}^{*}, \widetilde{N}_{\Delta t}^{*} ; \phi\right)=\left(u_{\Delta t}^{*}(0), \phi\right)_{L^{2}(D)}+\int_{0}^{t}\left\langle\operatorname{div}_{x}\left(\left|\nabla v_{\Delta t}\right|^{p-2} \nabla v_{\Delta t}(s)\right), \phi\right\rangle \mathrm{d} s \\
&+\int_{0}^{t}\left\langle\operatorname{div}_{x} \vec{f}\left(v_{\Delta t}(s)\right), \phi\right\rangle \mathrm{d} s+\left\langle b_{\Delta t}^{*}(t), \phi\right\rangle, \\
& \mathcal{K}_{*}\left(u_{*}, U_{*}, \widetilde{N}_{*} ; \phi\right)=\left(u_{*}(0), \phi\right)_{L^{2}(D)}+\int_{0}^{t}\left\langle\operatorname{div}_{x}\left(G(s)+\vec{f}\left(u_{*}(s)\right)\right), \phi\right\rangle \mathrm{d} s \\
&+\left\langle\int_{0}^{t} \int_{|z|>0} \eta\left(u_{*}(s-, \cdot) ; z\right) \widetilde{N}_{*}(\mathrm{~d} z, \mathrm{~d} s), \phi\right\rangle .
\end{aligned}
$$

In view of Lemma 3.11, we conclude that

$$
\lim _{\Delta t \rightarrow 0}\left\|\mathcal{K}_{\Delta t}^{*}\left(u_{\Delta t}^{*}, U_{\Delta t}^{*}, \widetilde{N}_{\Delta t}^{*} ; \phi\right)-\mathcal{K}_{*}\left(u_{*}, U_{*}, \widetilde{N}_{*} ; \phi\right)\right\|_{L^{1}(\bar{\Omega} \times[0, T])}=0
$$

Thanks to the definition of $\mathcal{K}_{\Delta t}\left(\tilde{u}_{\Delta t}, U, \tilde{N} ; \phi\right)$ and the equality (3.9), we have: $\overline{\mathbb{P}}$-a.s., $\left(\tilde{u}_{\Delta t}(t), \phi\right)_{L^{2}(D)}=\mathcal{K}_{\Delta t}\left(\tilde{u}_{\Delta t}, U, \widetilde{N} ; \phi\right)$ for all $t \in[0, T]$. More precisely,

$$
\overline{\mathbb{E}}\left[\int_{0}^{T}\left|\left(\tilde{u}_{\Delta t}(t), \phi\right)_{L^{2}(D)}-\mathcal{K}_{\Delta t}\left(\tilde{u}_{\Delta t}, \tilde{N} ; \phi\right)\right| \mathrm{d} t\right]=0 .
$$

Since $\mathcal{L}\left(u_{\Delta t}^{*}, U_{\Delta t}^{*}, N_{\Delta t}^{*}\right)=\mathcal{L}\left(\tilde{u}_{\Delta t}, U_{\Delta t}, N_{\Delta t}\right)$ with $N_{\Delta t}(\mathrm{~d} z, \mathrm{~d} t)=N(\mathrm{~d} z, \mathrm{~d} t)$ and $U_{\Delta t}=U$ for all $\Delta t>0$, we directly have

$$
\int_{0}^{T} \overline{\mathbb{E}}\left[\left|\left(u_{\Delta t}^{*}(t), \phi\right)_{L^{2}(D)}-\mathcal{K}_{\Delta t}^{*}\left(u_{\Delta t}^{*}, U_{\Delta t}^{*}, \widetilde{N}_{\Delta t}^{*} ; \phi\right)\right|\right] \mathrm{d} t=0 .
$$

Again, thanks to i) of Lemma 3.8, we see that

$$
\lim _{\Delta t \rightarrow 0}\left\|\left(u_{\Delta t}^{*}(\cdot), \phi\right)_{L^{2}(D)}-\left(u_{*}(\cdot), \phi\right)_{L^{2}(D)}\right\|_{L^{1}(\bar{\Omega} \times[0, T])}=0 .
$$

We combine (3.26)-(3.28) to conclude that $\overline{\mathbb{P}}$-a.s., for a.e. $t \in[0, T]$, and $\phi \in W_{0}^{1, p}(D)$

$$
\begin{array}{r}
\left(u_{*}(t), \phi\right)_{L^{2}(D)}=\left(u_{*}(0), \phi\right)_{L^{2}(D)}+\int_{0}^{t}\left\langle\operatorname{div}_{x}\left(G(s)+\vec{f}\left(u_{*}(s)\right)\right), \phi\right\rangle \mathrm{d} s \\
+\left\langle\int_{0}^{t} \int_{|z|>0} \eta\left(u_{*}(s-, \cdot) ; z\right) \widetilde{N}_{*}(\mathrm{~d} z, \mathrm{~d} s), \phi\right\rangle .
\end{array}
$$

Note that, $u_{\Delta t}^{*}(0)=u_{0, \Delta t}+U_{\Delta t}^{*}$ and $\overline{\mathbb{P}}$-a.s., $U_{\Delta t}^{*} \rightarrow U_{*}$ in $\mathbb{X}_{U}$. Since $u_{0, \Delta t} \rightarrow u_{0}$ in $L^{2}(D)$, by using i) of Lemma 3.8, we infer that $u_{*}(0)=u_{0}+U_{*}$. Hence, we obtain

$$
\begin{array}{r}
\left(u_{*}(t), \phi\right)_{L^{2}(D)}=\left(u_{0}+U_{*}, \phi\right)_{L^{2}(D)}+\int_{0}^{t}\left\langle\operatorname{div}_{x}\left(G(s)+\vec{f}\left(u_{*}(s)\right)\right), \phi\right\rangle \mathrm{d} s \\
+\left\langle\int_{0}^{t} \int_{|z|>0} \eta\left(u_{*}(s-, \cdot) ; z\right) \widetilde{N}_{*}(\mathrm{~d} z, \mathrm{~d} s), \phi\right\rangle .
\end{array}
$$


Since $u_{*} \in \mathbb{D}\left([0, T] ; L_{w}^{2}(D)\right)$, (3.29) holds true for all $t \in[0, T]$ and all $\phi \in W_{0}^{1, p}(D)$.

Step ii): We wish to identify the function $G \in L^{p^{\prime}}\left(\bar{\Omega} \times D_{T}\right)^{d}$. We take the $L^{2}$-scalar product with $v_{k+1}$ in (3.13) and use the identity $(a-b) a=\frac{1}{2}\left(|a|^{2}-|b|^{2}+|a-b|^{2}\right) \quad \forall a, b \in \mathbb{R}$ to have

$$
\begin{gathered}
\frac{1}{2} \overline{\mathbb{E}}\left[\left\|v_{k+1}\right\|_{L^{2}(D)}^{2}-\left\|v_{k}\right\|_{L^{2}(D)}^{2}+\left\|v_{k+1}-v_{k}\right\|_{L^{2}(D)}^{2}\right]+\Delta t \overline{\mathbb{E}}\left[\int_{D}\left|\nabla v_{k+1}\right|^{p-2} \nabla v_{k+1} \cdot \nabla v_{k+1} \mathrm{~d} x\right] \\
-\overline{\mathbb{E}}\left[\left(\int_{t_{k}}^{t_{k+1}} \int_{|z|>0} \eta\left(v_{k} ; z\right) \widetilde{N}_{\Delta t}^{*}(\mathrm{~d} z, \mathrm{~d} t), v_{k+1}-v_{k}\right)_{L^{2}(D)}\right]=0 .
\end{gathered}
$$

Since

$$
\begin{aligned}
-\overline{\mathbb{E}} & {\left[\left(\int_{t_{k}}^{t_{k+1}} \int_{|z|>0} \eta\left(v_{k} ; z\right) \widetilde{N}_{\Delta t}^{*}(\mathrm{~d} z, \mathrm{~d} t), v_{k+1}-v_{k}\right)_{L^{2}(D)}\right] } \\
=- & \frac{1}{2} \overline{\mathbb{E}}\left[\left\|v_{k+1}-v_{k}\right\|_{L^{2}(D)}^{2}\right]-\frac{1}{2} \overline{\mathbb{E}}\left[\left\|\int_{t_{k}}^{t_{k+1}} \int_{|z|>0} \eta\left(v_{k} ; z\right) \widetilde{N}_{\Delta t}^{*}(\mathrm{~d} z, \mathrm{~d} t)\right\|_{L^{2}(D)}^{2}\right] \\
& +\frac{1}{2} \overline{\mathbb{E}}\left[\left\|\int_{t_{k}}^{t_{k+1}} \int_{|z|>0} \eta\left(v_{k} ; z\right) \widetilde{N}_{\Delta t}^{*}(\mathrm{~d} z, \mathrm{~d} t)-\left(v_{K+1}-v_{k}\right)\right\|_{L^{2}(D)}^{2}\right],
\end{aligned}
$$

by summing over $k=0,1, \cdots, N-1$ in (3.30) and using the fact that $v_{N}=u_{\Delta t}^{*}(T)$, we get

$$
\begin{aligned}
& \frac{1}{2} \overline{\mathbb{E}}\left[\left\|u_{\Delta t}^{*}(T)\right\|_{L^{2}(D)}^{2}\right]+\overline{\mathbb{E}}\left[\int_{D_{T}}\left|\nabla v_{\Delta t}(t)\right|^{p-2} \nabla v_{\Delta t}(t) \cdot \nabla v_{\Delta t}(t) \mathrm{d} x \mathrm{~d} t\right] \\
& -\frac{1}{2} \sum_{k=0}^{N-1} \overline{\mathbb{E}}\left[\left\|\int_{t_{k}}^{t_{k+1}} \int_{|z|>0} \eta\left(v_{k} ; z\right) \widetilde{N}_{\Delta t}^{*}(\mathrm{~d} z, \mathrm{~d} t)\right\|_{L^{2}(D)}^{2}\right] \leq \frac{1}{2} \overline{\mathbb{E}}\left[\left\|u_{0, \Delta t}+U_{\Delta t}^{*}\right\|_{L^{2}(D)}^{2}\right] .
\end{aligned}
$$

Thanks to Itô-Lévy isometry, we see that

$$
\begin{aligned}
& \sum_{k=0}^{N-1} \overline{\mathbb{E}}\left[\left\|\int_{t_{k}}^{t_{k+1}} \int_{|z|>0} \eta\left(v_{k} ; z\right) \widetilde{N}_{\Delta t}^{*}(\mathrm{~d} z, \mathrm{~d} t)\right\|_{L^{2}(D)}^{2}\right] \\
& =\overline{\mathbb{E}}\left[\int_{0}^{T} \int_{|z|>0}\left\|\eta\left(\bar{v}_{\Delta t}(t) ; z\right)\right\|_{L^{2}(D)}^{2} m(\mathrm{~d} z) \mathrm{d} t\right] .
\end{aligned}
$$

Again, an application of Itô-Lévy formula [11, similar to Theorem 3.4] to the functional $\left\|u_{*}(t)\right\|_{2}^{2}$ in (3.29) yields

$$
\begin{aligned}
& \frac{1}{2} \overline{\mathbb{E}}\left[\left\|u_{*}(T)\right\|_{L^{2}(D)}^{2}\right]+\overline{\mathbb{E}}\left[\int_{D_{T}} G \cdot \nabla u_{*} \mathrm{~d} x \mathrm{~d} t\right]-\frac{1}{2} \overline{\mathbb{E}}\left[\int_{0}^{T} \int_{|z|>0}\left\|\eta\left(u_{*}(s-) ; z\right)\right\|_{L^{2}(D)}^{2} m(\mathrm{~d} z) \mathrm{d} s\right] \\
& =\frac{1}{2} \overline{\mathbb{E}}\left[\left\|u_{0}+U_{*}\right\|_{L^{2}(D)}^{2}\right] .
\end{aligned}
$$

Combining (3.31), (3.32), and (3.33) we obtain

$$
\begin{aligned}
& \frac{1}{2} \overline{\mathbb{E}}\left[\left\|u_{\Delta t}^{*}(T)\right\|_{L^{2}(D)}^{2}-\left\|u_{*}(T)\right\|_{L^{2}(D)}^{2}\right]+\overline{\mathbb{E}}\left[\int_{D_{T}}\left|\nabla v_{\Delta t}(t)\right|^{p-2} \nabla v_{\Delta t}(t) \cdot \nabla v_{\Delta t}(t) \mathrm{d} x \mathrm{~d} t\right] \\
& \quad-\frac{1}{2} \overline{\mathbb{E}}\left[\int_{0}^{T} \int_{|z|>0}\left(\left\|\eta\left(\bar{v}_{\Delta t}(s) ; z\right)\right\|_{L^{2}(D)}^{2}-\left\|\eta\left(u_{*}(s-) ; z\right)\right\|_{L^{2}(D)}^{2}\right) m(\mathrm{~d} z) \mathrm{d} s\right] \\
& \leq \overline{\mathbb{E}}\left[\int_{D_{T}} G \cdot \nabla u_{*} \mathrm{~d} x \mathrm{~d} t\right]+\frac{1}{2}\left\{\overline{\mathbb{E}}\left[\left\|u_{0, \Delta t}+U_{\Delta t}^{*}\right\|_{L^{2}(D)}^{2}\right]-\overline{\mathbb{E}}\left[\left\|u_{0}+U_{*}\right\|_{L^{2}(D)}^{2}\right]\right\} .
\end{aligned}
$$


Note that

$$
\left\{\begin{array}{l}
\liminf _{\Delta t>0} \overline{\mathbb{E}}\left[\left\|u_{\Delta t}^{*}(T)\right\|_{L^{2}(D)}^{2}-\left\|u_{*}(T)\right\|_{L^{2}(D)}^{2}\right] \geq 0 \\
\overline{\mathbb{E}}\left[\left\|u_{0, \Delta t}+U_{\Delta t}^{*}\right\|_{L^{2}(D)}^{2}\right] \rightarrow \overline{\mathbb{E}}\left[\left\|u_{0}+U_{*}\right\|_{L^{2}(D)}^{2}\right]
\end{array}\right.
$$

and thanks to ii) of Lemma 3.8 along with the assumptions $\mathbf{A . 3}$ and $\mathbf{\text { A.4. }}$, it follows that

$$
\overline{\mathbb{E}}\left[\int_{0}^{T} \int_{|z|>0}\left\|\eta\left(\bar{v}_{\Delta t}(t) ; z\right)\right\|_{L^{2}(D)}^{2} m(\mathrm{~d} z) \mathrm{d} t\right] \rightarrow \overline{\mathbb{E}}\left[\int_{0}^{T} \int_{|z|>0}\left\|\eta\left(u_{*}(t-) ; z\right)\right\|_{L^{2}(D)}^{2} m(\mathrm{~d} z) \mathrm{d} t\right] .
$$

Thus, one arrives at the following inequality

$$
\limsup _{\Delta t>0} \overline{\mathbb{E}}\left[\int_{D_{T}}\left|\nabla v_{\Delta t}(t)\right|^{p-2} \nabla v_{\Delta t}(t) \cdot \nabla v_{\Delta t}(t) \mathrm{d} x \mathrm{~d} t\right] \leq \overline{\mathbb{E}}\left[\int_{D_{T}} G \cdot \nabla u_{*} \mathrm{~d} x \mathrm{~d} t\right] .
$$

We recall that $\nabla v_{\Delta t} \rightarrow \nabla u_{*}$ in $L^{p}\left(\bar{\Omega} \times D_{T}\right)^{d}$ and $\left|\nabla v_{\Delta t}\right|^{p-2} \nabla v_{\Delta t} \rightarrow G$ in $L^{p^{\prime}}\left(\bar{\Omega} \times D_{T}\right)^{d}$. Since $p>2$, there exists a constant $C>0$, independent of $\Delta t$, such that

$$
\begin{aligned}
& C \limsup _{\Delta t \rightarrow 0} \overline{\mathbb{E}}\left[\int_{D_{T}}\left|\nabla v_{\Delta t}-\nabla u_{*}\right|^{p} \mathrm{~d} x \mathrm{~d} t\right] \\
& \leq \limsup _{\Delta t \rightarrow 0} \overline{\mathbb{E}}\left[\int_{D_{T}}\left(\left|\nabla v_{\Delta t}\right|^{p-2} \nabla v_{\Delta t}-\left|\nabla u_{*}\right|^{p-2} \nabla u_{*}\right) \cdot \nabla\left(v_{\Delta t}-u_{*}\right) \mathrm{d} x \mathrm{~d} t\right] \\
& \leq \underset{\Delta t>0}{\limsup } \overline{\mathbb{E}}\left[\int_{D_{T}}\left|\nabla v_{\Delta t}(t)\right|^{p-2} \nabla v_{\Delta t}(t) \cdot \nabla v_{\Delta t}(t) \mathrm{d} x \mathrm{~d} t\right]-\overline{\mathbb{E}}\left[\int_{D_{T}} G \cdot \nabla u_{*} \mathrm{~d} x \mathrm{~d} t\right] \leq 0,
\end{aligned}
$$

where the last inequality follows from (3.34). Therefore, since $\nabla v_{\Delta t} \rightarrow \nabla u_{*}$ in $L^{p}\left(\bar{\Omega} \times D_{T}\right)^{d}$ we conclude that $\nabla v_{\Delta t} \rightarrow \nabla u_{*}$ in $L^{p}\left(\bar{\Omega} \times D_{T}\right)^{d}$, and hence $\left|\nabla v_{\Delta t}\right|^{p-2} \nabla v_{\Delta t} \rightarrow\left|\nabla u_{*}\right|^{p-2} \nabla u_{*}$ in $L^{p^{\prime}}\left(\bar{\Omega} \times D_{T}\right)^{d}$. In other words, $G=\left|\nabla u_{*}\right|^{p-2} \nabla u_{*}$.

Step iii): With the identification of $G$, it follows from (3.29) that the system $\bar{\pi}:=\left(\bar{\Omega}, \overline{\mathcal{F}}, \overline{\mathbb{P}}, \overline{\mathbb{F}}, N_{*}, u_{*}, U_{*}\right)$ is a weak solution of the problem (1.1). Moreover, since

$$
\mathcal{L}\left(U_{\Delta t}^{*}\right)=\mathcal{L}\left(U_{\Delta t}\right) \text { on } W^{1, p}(D) \text { with } U_{\Delta t}=U \text {, and } \mathbb{P} \text {-a.s., } U_{\Delta t}^{*} \rightarrow U_{*} \text { in } \mathbb{X}_{U},
$$

we see that i) in Theorem 2.1 holds. Furthermore, one can use Proposition 3.7 and the estimates (3.15)-(3.16) to arrive at ii), Theorem 2.1. This completes the existence proof.

3.5.1. On path-wise uniqueness of weak solutions: Let $\left(\Omega, \mathcal{F}, \mathbb{P}, \mathbb{F}, N, u_{1}, U\right)$ and $\left(\Omega, \mathcal{F}, \mathbb{P}, \mathbb{F}, N, u_{2}, U\right)$ be two weak solutions of (1.1) with a given control $U$. Let us introduce the convex approximation of the absolute value function. Let $\beta: \mathbb{R} \rightarrow \mathbb{R}$ be a $C^{\infty}$ function satisfying

$$
\beta(0)=0, \quad \beta(-r)=\beta(r), \quad \beta^{\prime}(-r)=-\beta^{\prime}(r), \quad \beta^{\prime \prime} \geq 0,
$$

and

$$
\beta^{\prime}(r)=\left\{\begin{array}{l}
-1 \quad \text { when } r \leq-1 \\
\in[-1,1] \quad \text { when }|r|<1 \\
+1 \quad \text { when } r \geq 1
\end{array}\right.
$$

For any $\vartheta>0$, define $\beta_{\vartheta}: \mathbb{R} \rightarrow \mathbb{R}$ by $\beta_{\vartheta}(r)=\vartheta \beta\left(\frac{r}{\vartheta}\right)$. Then

$$
|r|-M_{1} \vartheta \leq \beta_{\vartheta}(r) \leq|r| \quad \text { and } \quad\left|\beta_{\vartheta}^{\prime \prime}(r)\right| \leq \frac{M_{2}}{\vartheta} \mathbf{1}_{|r| \leq \vartheta},
$$

where $M_{1}=\sup _{|r| \leq 1}|| r|-\beta(r)|$ and $M_{2}=\sup _{|r| \leq 1}\left|\beta^{\prime \prime}(r)\right|$.

We apply Itô-Lévy formula to the functional $\int_{D} \beta_{\vartheta}\left(u_{1}(t)-u_{2}(t)\right) \mathrm{d} x$ and have

$$
\int_{D} \beta_{\vartheta}\left(u_{1}(t)-u_{2}(t)\right) \mathrm{d} x
$$




$$
\begin{aligned}
& =-\int_{0}^{t} \int_{D}\left(\left|\nabla u_{1}\right|^{p-2} \nabla u_{1}-\left|\nabla u_{2}\right|^{p-2} \nabla u_{2}\right) \cdot \nabla\left(u_{1}-u_{2}\right)(s) \beta_{\vartheta}^{\prime \prime}\left(u_{1}-u_{2}\right) \mathrm{d} x \mathrm{~d} s \\
& -\int_{0}^{t} \int_{D}\left(\vec{f}\left(u_{1}(s, x)\right)-\vec{f}\left(u_{2}(s, x)\right)\right) \cdot \nabla\left(u_{1}(s, x)-u_{2}(s, x)\right) \beta_{\vartheta}^{\prime \prime}\left(u_{1}-u_{2}\right) \mathrm{d} x \mathrm{~d} s \\
& +\int_{0}^{t} \int_{|z|>0} \int_{D} \int_{0}^{1} \beta_{\vartheta}^{\prime}\left(\left(u_{1}-u_{2}\right)(s-, x)+\lambda\left(\eta\left(u_{1}(s-, x) ; z\right)-\eta\left(u_{2}(s-, x) ; z\right)\right)\right) \\
& \times \int_{0}^{t} \int_{|z|>0} \int_{D} \int_{0}^{1}(1-\lambda) \beta_{\vartheta}^{\prime \prime}\left(\left(u_{1}(s-, x) ; z\right)-\eta\left(u_{2}(s-, x) ; z\right)\right) \mathrm{d} \lambda \mathrm{d} x \widetilde{N}(\mathrm{~d} z, \mathrm{~d} s) \\
& \times\left(\eta\left(u_{1}(s-, x) ; z\right)-\eta\left(u_{2}(s-, x) ; z\right)\right)^{2} \mathrm{~d} \lambda \mathrm{d} x m(\mathrm{~d} z) \mathrm{d} s .
\end{aligned}
$$

Since $p>2$ and $\beta_{\vartheta}^{\prime \prime} \geq 0$, we see that

$$
\begin{aligned}
& -\left(\left|\nabla u_{1}\right|^{p-2} \nabla u_{1}-\left|\nabla u_{2}\right|^{p-2} \nabla u_{2}\right) \cdot \nabla\left(u_{1}-u_{2}\right) \beta_{\vartheta}^{\prime \prime}\left(u_{1}-u_{2}\right) \\
& \leq-C\left|\nabla\left(u_{1}-u_{2}\right)\right|^{p} \beta_{\vartheta}^{\prime \prime}\left(u_{1}-u_{2}\right) \leq 0,
\end{aligned}
$$

and therefore, we obtain

$$
\begin{aligned}
& \mathbb{E}\left[\int_{D} \beta_{\vartheta}\left(u_{1}(t)-u_{2}(t)\right) \mathrm{d} x\right] \\
& \leq \mathbb{E}\left[-\int_{0}^{t} \int_{D}\left(\vec{f}\left(u_{1}(s, x)\right)-\vec{f}\left(u_{2}(s, x)\right) \cdot \nabla\left(u_{1}(s, x)-u_{2}(s, x)\right) \beta_{\vartheta}^{\prime \prime}\left(u_{1}-u_{2}\right) \mathrm{d} x \mathrm{~d} s\right]\right. \\
& +\mathbb{E}\left[\int_{0}^{t} \int_{|z|>0} \int_{D} \int_{0}^{1}(1-\lambda) \beta_{\vartheta}^{\prime \prime}\left(u_{1}(s, x)-u_{2}(s, x)+\lambda\left(\eta\left(u_{1}(s, x) ; z\right)-\eta\left(u_{2}(s, x) ; z\right)\right)\right)\right. \\
& \left.\times\left(\eta\left(u_{1}(s, x) ; z\right)-\eta\left(u_{2}(s, x) ; z\right)\right)^{2} \mathrm{~d} \lambda \mathrm{d} x m(\mathrm{~d} z) \mathrm{d} s\right] \\
& \equiv \mathcal{A}+\mathcal{B} .
\end{aligned}
$$

Since $\beta_{\vartheta}^{\prime \prime}(r) \leq \frac{M_{2}}{\vartheta} \mathbf{1}_{\{|r| \leq \vartheta\}}$ and $\vec{f}$ is a Lipschitz continuous function, we have $\mathbb{P}$-a.s.,

$$
\begin{aligned}
& \left(\vec{f}\left(u_{1}\right)-\vec{f}\left(u_{2}\right)\right) \cdot \nabla\left(u_{1}(s, x)-u_{2}(s, x)\right) \beta_{\vartheta}^{\prime \prime}\left(u_{1}-u_{2}\right) \\
& \leq c_{f}\left|u_{1}-u_{2}\right|\left|\nabla\left(u_{1}-u_{2}\right)\right| \frac{M_{2}}{\vartheta} \mathbf{1}_{\left\{\left|u_{1}-u_{2}\right| \leq \vartheta\right\}} \rightarrow 0 \quad(\vartheta \rightarrow 0)
\end{aligned}
$$

for almost every $(t, x) \in D_{T}$. Moreover

$$
\left|\vec{f}\left(u_{1}\right)-\vec{f}\left(u_{2}\right)\right| \mid \nabla\left(u_{1}(s, x)-u_{2}(s, x)\left|\beta_{\vartheta}^{\prime \prime}\left(u_{1}-u_{2}\right) \leq M_{2}\right| \nabla\left(u_{1}-u_{2}\right) \mid \in L^{1}\left(\Omega \times D_{T}\right) .\right.
$$

Thus, by dominated convergence theorem we conclude that $\mathcal{A} \rightarrow 0$ as $\vartheta \rightarrow 0$.

Next we move on to estimate $\mathcal{B}$. Let

$$
a=u_{1}(s, x)-u_{2}(s, x) \quad \text { and } \quad b=\eta\left(u_{1}(s, x) ; z\right)-\eta\left(u_{2}(s, x) ; z\right) .
$$

Then, we have, in view of the assumption A.3.

$$
\begin{aligned}
\mathcal{B} & =\mathbb{E}\left[\int_{0}^{t} \int_{|z|>0} \int_{D} \int_{0}^{1}(1-\lambda) b^{2} \beta_{\vartheta}^{\prime \prime}(a+\lambda b) \mathrm{d} \lambda \mathrm{d} x m(\mathrm{~d} z) \mathrm{d} s\right] \\
& \leq \mathbb{E}\left[\int_{0}^{t} \int_{|z|>0} \int_{D} \int_{0}^{1}(1-\lambda) a^{2} \beta_{\vartheta}^{\prime \prime}(a+\lambda b)\left(1 \wedge|z|^{2}\right) \mathrm{d} \lambda \mathrm{d} x m(\mathrm{~d} z) \mathrm{d} s\right] .
\end{aligned}
$$

Note that $\beta_{\vartheta}^{\prime \prime}$ is non-negative and symmetric around zero. Thus we may assume, without loss of generality, that $a \geq 0$. Then by the assumption A.3

$$
u_{1}(s, x)-u_{2}(s, x)+\lambda b \geq\left(1-\lambda^{*}\right)\left(u_{1}(s, x)-u_{2}(s, x)\right)
$$


for $\lambda \in[0,1]$. In other words

$$
0 \leq a \leq\left(1-\lambda^{*}\right)^{-1}(a+\lambda b)
$$

We combine (3.37) and (3.38) to obtain

$$
\mathcal{B} \leq C\left(\lambda^{*}\right) \mathbb{E}\left[\int_{0}^{t} \int_{|z|>0} \int_{D} \int_{0}^{1}(1-\lambda)(a+\lambda b)^{2} \beta_{\vartheta}^{\prime \prime}(a+\lambda b)\left(1 \wedge|z|^{2}\right) \mathrm{d} \lambda \mathrm{d} x m(\mathrm{~d} z) \mathrm{d} s\right] .
$$

In view of (3.35), and the assumption on $\eta$ that $\eta(0, z)=0$ for all $z \in \mathbb{R}$, we see that for each $\lambda \in[0,1]$

$$
(a+\lambda b)^{2} \beta_{\vartheta}^{\prime \prime}(a+\lambda b) \leq|a+\lambda b| \mathbf{1}_{\{0<|a+\lambda b|<\vartheta\}} \leq|a+\lambda b| \in L^{1}\left(\Omega \times D_{T}\right)
$$

for $m(\mathrm{~d} z)$-almost every $z \in \mathbb{R}$. Again $|a+\lambda b| \mathbf{1}_{\{0<|a+\lambda b|<\vartheta\}} \rightarrow 0$ as $\vartheta \rightarrow 0$ for almost every $(s, x)$ and almost surely. We apply dominated convergence theorem, along with the assumption A.4 to conclude that $\mathcal{B} \rightarrow 0$ as $\vartheta \rightarrow 0$. Putting things together and passing to the limit in (3.36), we have

$$
\mathbb{E}\left[\int_{D}\left|u_{1}(t, x)-u_{2}(t, x)\right| \mathrm{d} x\right]=0 .
$$

In other words, $\mathbb{P}$-a.s., $u_{1}(t, x)=u_{2}(t, x)$ for almost every $(t, x)$. This yields the uniqueness of path-wise weak solution of the underlying problem (1.1) with respect to the same stochastic basis. This completes the proof of Theorem 2.1.

Remark 3.1. Existence of weak solution and path-wise uniqueness guarantee uniqueness in law due to classical Yamada-Watanabe technique [30] for finite dimensional case; for the infinite dimensional case, see e.g., [22, Theorems 2 and 11].

Remark 3.2. Thanks to Skorokhod parameterization, see e.g., [3, 10, one can prove the following theorem as a generalization of Theorem 2.1 .

Let the assumptions $\mathbf{A}$.1 $\mathbf{A} .4$ be true and $\left(\Omega, \mathcal{F}, \mathbb{P},\left\{\mathcal{F}_{t}\right\}\right)$ be a given filtered probability space satisfying the usual hypotheses. Let $N$ be a time-homogeneous Poisson random measure on $\mathbb{R}$ with intensity measure $m(d z)$ defined on $\left(\Omega, \mathcal{F}, \mathbb{P},\left\{\mathcal{F}_{t}\right\}\right)$, and $\mu$ be a probability measure on $L^{2}(D)$ such that $\int_{L^{2}(D)} \Phi(v) \mu(\mathrm{d} v)<\infty$ where $\Phi(v)=\|v\|_{W^{1, p}(D)}^{p}$. Then, for the problem (1.1), there exists a weak solution $\hat{\pi}=\left(\hat{\Omega}, \hat{\mathcal{F}}, \hat{\mathbb{P}},\left\{\hat{\mathcal{F}}_{t}\right\}, \hat{N}, \hat{u}, \hat{U}\right)$ in the sense of Definition 2.1] such that (2.1) holds and $\mu=\mathcal{L}(\hat{U})$ on $L^{2}(D)$.

\section{Existence of optimal Control: Proof of Theorem 2.2}

The objective of this section is to prove existence of a weak optimal solution of (1.2) in the sense of Definition 2.2 i.e., Theorem 2.2 ,

Proof. We prove Theorem 2.2 in several steps.

Step I): In view of Theorem 2.1, there exists a weak solution of (1.1) with $U=0$, and satisfies the estimate ii) of Theorem 2.1. Since $\Psi$ is Lipschitz continuous and $u_{\operatorname{tar}} \in L^{p}\left(0, T ; W_{0}^{1, p}(D)\right)$, $\Lambda$ is finite. Thus, there exists a minimizing sequence of weak admissible solutions $\pi_{n}=\left(\Omega_{n}, \mathcal{F}_{n}, \mathbb{P}_{n}, \mathbb{F}_{n}=\left\{\mathcal{F}_{t}^{n}\right\}, N_{n}, u_{n}, U_{n}\right)$ such that $\Lambda=\lim _{n \rightarrow \infty} \mathcal{J}\left(\pi_{n}\right)$. Since for each $n \in \mathbb{N}$, $\pi_{n} \in \mathcal{U}_{\mathrm{ad}}^{w}\left(u_{0} ; T\right)$, we have, $\mathbb{P}_{n}$-a.s. for all $t \in[0, T]$

$$
\begin{aligned}
u_{n}(t) & =u_{0}+U_{n}+\int_{0}^{t} \operatorname{div}_{x}\left(\left|\nabla u_{n}\right|^{p-2} \nabla u_{n}+\vec{f}\left(u_{n}\right)\right) \mathrm{d} s+\int_{0}^{t} \int_{|z|>0} \eta\left(u_{n} ; z\right) \widetilde{N}_{n}(\mathrm{~d} z, \mathrm{~d} s) \\
& =u_{0}+U_{n}+T_{1, n}(t)+T_{2, n}(t) .
\end{aligned}
$$


Moreover, since $\Lambda$ is finite, one has the following estimates (uniform in $n$ ):

$$
\left\{\begin{array}{l}
\sup _{n} \mathbb{E}_{n}\left[\left\|U_{n}\right\|_{W^{1, p}(D)}^{p}\right] \leq C, \\
\sup _{n} \mathbb{E}_{n}\left[\sup _{0 \leq t \leq T}\left\|u_{n}(t)\right\|_{L^{2}(D)}^{2}+\int_{0}^{T}\left\|u_{n}(t)\right\|_{W_{0}^{1, p}(D)}^{p} \mathrm{~d} t\right] \leq C,
\end{array}\right.
$$

where $\mathbb{E}_{n}$ denotes the expectation with respect to $\mathbb{P}_{n}$.

Step II): By proving Aldous condition for the sequence $\left\{u_{n}\right\}$ in $W^{-1, p^{\prime}}(D)$ and then applying Theorem 3.4 along with the uniform-estimate (4.2), one can establish the tightness of $\left\{\mathcal{L}\left(u_{n}\right)\right\}$ on $(\mathcal{Z}, \mathcal{T})$. Moreover, due to the uniform-bound (4.2), and the tightness of the family of laws $\left\{\mathcal{L}\left(N_{n}(\mathrm{~d} z, \mathrm{~d} t)\right)\right\}$ on $\left.M_{\overline{\mathbb{N}}} \mathbb{R} \times[0, T]\right)$, the set $\left\{\mathcal{L}\left(u_{n}, N_{n}, U_{n}\right)\right\}$ is tight in $\mathcal{X}$. Therefore, by [19, Corollary 2], there exist a subsequence of $\{n\}$, still we denote it by same $\{n\}$, a probability space $\left(\Omega^{*}, \mathcal{F}^{*}, \mathbb{P}^{*}\right)$ and, on this space $\mathcal{X}$-valued random variables $\left(u^{*}, U^{*}, N^{*}\right)$ and $\left(u_{n}^{*}, U_{n}^{*}, N_{n}^{*}\right)$ such that

i). $\mathcal{L}\left(u_{n}^{*}, U_{n}^{*}, N_{n}^{*}\right)=\mathcal{L}\left(u_{n}, U_{n}, N_{n}\right)$ for all $n \in \mathbb{N}$,

ii). $\left(u_{n}^{*}, U_{n}^{*}, N_{n}^{*}\right) \rightarrow\left(u^{*}, U^{*}, N^{*}\right)$ in $\mathcal{X} \mathbb{P}^{*}$-a.s. $(n \rightarrow \infty)$,

iii). $N_{n}^{*}\left(\omega^{*}\right)=N *\left(\omega^{*}\right)$ for all $\omega^{*} \in \Omega^{*}$.

The sequences $\left\{u_{n}^{*}\right\}$ and $\left\{U_{n}^{*}\right\}$ satisfy the same estimate as the original sequences $\left\{u_{n}\right\}$ and $\left\{U_{n}\right\}$ respectively. In particular,

$$
\left\{\begin{array}{l}
\sup _{n} \mathbb{E}^{*}\left[\left\|U_{n}^{*}\right\|_{W^{1, p}(D)}^{p}\right] \leq C, \\
\sup _{n} \mathbb{E}^{*}\left[\sup _{0 \leq t \leq T}\left\|u_{n}^{*}(t)\right\|_{L^{2}(D)}^{2}+\int_{0}^{T}\left\|u_{n}^{*}(t)\right\|_{W_{0}^{1, p}(D)}^{p} \mathrm{~d} t\right] \leq C .
\end{array}\right.
$$

Moreover, in view of (4.1) and i) of Step II, one can conclude $\mathbb{P}^{*}$-a.s.,

$$
\begin{gathered}
u_{n}^{*}(t)=u_{0}+U_{n}^{*}+\int_{0}^{t} \operatorname{div}_{x}\left(\left|\nabla u_{n}^{*}(s)\right|^{p-2} \nabla u_{n}^{*}(s)+\vec{f}\left(u_{n}^{*}(s)\right)\right) \mathrm{d} s \\
+\int_{0}^{t} \int_{|z|>0} \eta\left(u_{n}^{*}(s) ; z\right) \widetilde{N}_{n}^{*}(\mathrm{~d} z, \mathrm{~d} s) .
\end{gathered}
$$

Step III): Let $\mathbb{F}^{*}$ be the natural filtration of $\left(u_{n}^{*}, N_{n}^{*}, u^{*}, N^{*}\right)$. Since $N_{n}^{*}\left(\omega^{*}\right)=N^{*}\left(\omega^{*}\right)$ for all $\omega^{*} \in \Omega^{*}, N_{n}^{*}$ and $N^{*}$ are the time homogeneous Poisson random measures on $\mathbb{R}$ over the stochastic basis $\left(\Omega^{*}, \mathcal{F}^{*}, \mathbb{P}^{*}, \mathbb{F}^{*}\right)$. Using the similar arguments as in Lemmas 3.8 - 3.9 and 3.11 along with step ii) in subsection 3.5. one can pass to the limit in (4.4) and conclude that the $W_{0}^{1, p}(D)$-valued $\mathbb{F}^{*}$-predictable stochastic process $u^{*}$ satisfies the following: $\mathbb{P}^{*}$-a.s. and a.e. $t \in[0, T]$,

$$
\begin{array}{r}
\left(u^{*}(t), \phi\right)_{L^{2}(D)}=\left(u_{0}+U^{*}, \phi\right)_{L^{2}(D)}+\int_{0}^{t}\left\langle\operatorname{div}_{x}\left(\left|\nabla u^{*}(s)\right|^{p-2} \nabla u^{*}(s)+\vec{f}\left(u^{*}(s)\right)\right), \phi\right\rangle \mathrm{d} s \\
+\left\langle\int_{0}^{t} \int_{|z|>0} \eta\left(u^{*}(s) ; z\right) \widetilde{N}^{*}(\mathrm{~d} z, \mathrm{~d} s), \phi\right\rangle \quad \forall \phi \in W_{0}^{1, p}(D) .
\end{array}
$$

Since $u^{*} \in \mathbb{D}\left([0, T] ; L_{w}^{2}(D)\right)$, (4.5) holds true for all $t \in[0, T]$ and all $\phi \in W_{0}^{1, p}(D)$, and hence $\pi^{*}=\left(\Omega^{*}, \mathcal{F}^{*}, \mathbb{P}^{*}, \mathbb{F}^{*}, N^{*}, u^{*}, U^{*}\right) \in \mathcal{U}_{\mathrm{ad}}^{w}\left(u_{0} ; T\right)$. Moreover, $\left(u^{*}, U^{*}\right)$ satisfies the estimate (2.1).

Step IV): Since $\pi^{*} \in \mathcal{U}_{\mathrm{ad}}^{w}\left(u_{0} ; T\right)$, obviously $\Lambda \leq \mathcal{J}\left(\pi^{*}\right)$. We now show that $\mathcal{J}\left(\pi^{*}\right) \leq \Lambda$. Note that, the mapping

$$
\begin{aligned}
S: L^{2}(D) \times W^{1, p}(D) & \mapsto[0, \infty] \\
(u, U) & \mapsto\left\|u-u_{\operatorname{tar}}\right\|_{L^{2}(D)}^{2}+\|U\|_{W^{1, p}(D)}^{p}
\end{aligned}
$$


is a measurable, non-negative and lower semi-continuous convex function. Thus, invoking i)-iii) of step II along with Fatou's lemma, we get

$$
\begin{aligned}
\mathcal{J}\left(\pi^{*}\right) & =\mathbb{E}^{*}\left[\int_{0}^{T}\left\|u^{*}(t)-u_{\operatorname{tar}}(t)\right\|_{L^{2}(D)}^{2} \mathrm{~d} t+\left\|U^{*}\right\|_{W^{1, p}(D)}^{p}\right]+\mathbb{E}^{*}\left[\Psi\left(u^{*}(T)\right)\right] \\
& \leq \liminf _{n \rightarrow \infty}\left\{\mathbb{E}^{*}\left[\int_{0}^{T}\left\|u_{n}^{*}(t)-u_{\operatorname{tar}}(t)\right\|_{L^{2}(D)}^{2} \mathrm{~d} t+\left\|U_{n}^{*}\right\|_{W^{1, p}(D)}^{p}\right]+\mathbb{E}^{*}\left[\Psi\left(u_{n}^{*}(T)\right)\right]\right\} \\
& =\liminf _{n \rightarrow \infty}\left\{\mathbb{E}_{n}\left[\int_{0}^{T}\left\|u_{n}(t)-u_{\operatorname{tar}}(t)\right\|_{L^{2}(D)}^{2} \mathrm{~d} t+\left\|U_{n}\right\|_{W^{1, p}(D)}^{p}\right]+\mathbb{E}_{n}\left[\Psi\left(u_{n}(T)\right)\right]\right\} \\
& =\liminf _{n \rightarrow \infty} \mathcal{J}\left(\pi_{n}\right)=\Lambda .
\end{aligned}
$$

This implies that $\pi^{*}=\left(\Omega^{*}, \mathcal{F}^{*}, \mathbb{P}^{*}, \mathbb{F}^{*}, N^{*}, u^{*}, U^{*}\right)$ is a weak optimal solution of the control problem (1.2), and $U^{*}$ is an optimal control. This finishes the proof of Theorem 2.2.

Acknowledgements: The author would like to acknowledge the financial support by Department of Science and Technology, Govt. of India-the INSPIRE fellowship (IFA18-MA119).

\section{REFERENCES}

[1] P. Billingsley. Convergence of Probability measures. Second edition. Wiley Series in Probability and Statistics: Probability and Statistics. A Wiley-Interscience Publication. John Wiley \& Sons, Inc., New York, 1999.

[2] I. H. Biswas, K. H. Karlsen, A. K. Majee. Conservation laws driven by Lévy white noise. J. Hyperb. Diff. Equ. 12 (3) (2015) 581-654.

[3] D. Blackwell, L. E. Dubins. An extension of Skorokhods almost sure representation theorem. Proc. Am. Math. Soc. 89(4), 691-692, 1983.

[4] Z. Brzeźniak and E. Hausenblas. Maximal regularity for stochastic convolutions driven by Lévy processes. Probab. Theory Relat. Fields (2009) 145:615-637.

[5] Z. Brzeźniak, E. Hausenblas and P. A. Razafimandimby. Stochastic reaction diffusion equation driven by jump processes. Potential Anal. 49 (2018), no. 1, 131-201.

[6] Z. Brzeźniak and E. Motyl. Existence of a martingale solution of the stochastic Navier-Stokes equations in unbounded $2 D$ and $3 D$ domains. Journal of Differential Equations, (2013) 1627-1685.

[7] Z. Brzeźniak, R. Serrano. Optimal relaxed control of dissipative stochastic partial differential equations in Banach spaces. SIAM J. Control Optim. 51(3), 2664-2703, 2013.

[8] E. Dibenedetto. Degenerate parabolic equations. Springer-Verlag, New York, 1993.

[9] T. Dunst, A. K. Majee, A. Prohl, and G. Vallet. On Stochastic Optimal Control in Ferromagnetism. Arch. Rational Mech. Anal. (DOI) https://doi.org/10.1007/s00205-019-01381-w.

[10] X. Fernique. Un modèle presque sûr pour la convergence en loi. (French) [An almost sure model for weak convergence]. C. R. Acad. Sci. Paris Sér. I Math. 306(7), 335-338, 1988.

[11] W. Grecksch and C. Tudor. Stochastic Evolution Equations. A Hilbert space approach. Mathematical Research, 85. Akademie-Verlag, Berlin, 1995.

[12] I. Gyöngy, N. Krylov. Existence of strong solutions for Itô's stochastic equations via approximations. Probab. Theory Relat. Fields 105, 143-158 (1996).

[13] N. Ikeda, S. Watanabe. Stochastic Differential Equations and Diffusion Processes. NorthHolland Publishing Company, Amsterdam (1981).

[14] A. Jakubowski. The almost sure Skorokhod representation for subsequences in nonmetric spaces. Theory Probab. Appl. Vol. 42, No. 1 (1998) 164-174.

[15] W. Liu and M. Röckner. Stochastic partial differential equations: an introduction. Universitext. Springer, Cham, 2015.

[16] M. Métivier. Stochastic partial differential equations in infinite dimensional spaces. Scuola Normale Superiore, Pisa (1988). 
[17] M. Métivier, M. Viot. On weak solutions of stochastic partial differential equations. Lect. Notes Math. 1322/1988, 139-150 (1988).

[18] R. Mikulevicius and B. L. Rozovskii. Global $L_{2}$-solutions of stochastic Navier-Stokes equations. Ann. Probab. 33 (1) (2005) 137-176.

[19] E. Motyl. Stochastic Navier-Stokes Equations Driven by Lévy Noise in Unbounded 3D Domains. Potential Anal. (2013) 38:863-912.

[20] E. Nabana. Uniqueness for positive solutions of $p$-Laplacian problem in an annulus. Ann. Fac. Sci. Toulouse Math., 8 (1999).

[21] N. Nagase, M. Nisio. Optimal controls for stochastic partial differential equations. SIAM. Control Optim. 28(1), 186-213, 1990.

[22] M. Ondreját. Uniqueness for stochastic evolution equations in Banach spaces. Dissertationes Mathematicae, 426, pp. 1-63 (2004).

[23] E. Pardoux. Équations aux dérivées partielles stochastiques non linéaires monotones. PhD thesis, University of Paris Sud 1975.

[24] K. R. Parthasarathy. Probability measures on metric spaces. Academic Press, New York and London, 1967.

[25] S. Peszat and J. Zabczyk. Stochastic partial differential equations with Lévy noise, volume 113 of Encyclopedia of Mathematics and its Applications. Cambridge University Press, Cambridge, 2007. An evolution equation approach.

[26] T. Roubíček. Nonlinear Partial Differential Equations with Applications. Springer, Basel 2013.

[27] A. V. Skorokhod. Limit theorems for stochastic processes. Th. Probab. Appl., 1 (1956) pp. 261-290.

[28] G. Vallet, A. Zimmermann. Well-posedness for a pseudomonotone evolution problem with multiplicative noise. J. Evol. Equ. 19(2019), 153-202.

[29] A. W. Van der Vaart, Jon A. Wellner. Weak convergence and empirical processes with applications to statistics. Springer Series in Statistics. Springer-Verlag, New York, 1996.

[30] S. Watanabe, T. Yamada. On the uniqueness of solutions of stochastic differential equations. II. J. Math. Kyoto Univ. 11, pp. 155-167 (1971).

[31] Z. Wu, J. Zhao, J. Yin, H. Li. Nonlinear diffusion equations. World Scientific Publising, 2001.

[32] J. N. Zhao. On the Cauchy problem and initial traces for the evolution $p$-laplacian equation with strongly nonlinear sources. J. Diff. Equ., 121 (1995), 329-383.

(Ananta K. Majee)

Department of Mathematics, Indian Institute of Technology Delhi, Hauz Khas, New Delhi-110016, INDIA.

E-mail address: majee@maths.iitd.ac.in 